\title{
Improving the signal detection accuracy of functional Magnetic Resonance Imaging
}

\author{
Niels Janssen ${ }^{\mathrm{a}, \mathrm{b}, *}$, Juan A. Hernández-Cabrera ${ }^{\mathrm{a}, \mathrm{c}}$, Laura Ezama Foronda ${ }^{\mathrm{a}}$ \\ ${ }^{a}$ Psychology Department, Universidad de la Laguna, Tenerife, Spain \\ ${ }^{b}$ Institute of Biomedical Technologies, Universidad de la Laguna, Tenerife, Spain \\ ${ }^{c}$ Basque Center on Cognition, Brain and Language, San Sebastián, Spain
}

\begin{abstract}
A major drawback of functional Magnetic Resonance Imaging (fMRI) concerns the lack of detection accuracy of the measured signal. Although this limitation stems in part from the neuro-vascular nature of the fMRI signal, it also reflects particular methodological decisions in the fMRI data analysis pathway. Here we show that the signal detection accuracy of fMRI is affected by the specific way in which whole-brain volumes are created from individually acquired brain slices, and by the method of statistically extracting signals from the sampled data. To address these limitations, we propose a new framework for fMRI data analysis. The new framework creates whole-brain volumes from individual brain slices that are all acquired at the same point in time relative to a presented stimulus. These wholebrain volumes contain minimal temporal distortions, and are available at a high temporal resolution. In addition, statistical signal extraction occurred on the basis of a non-standard time point-by-time point approach. We evaluated the detection accuracy of the extracted signal in the standard and new framework with simulated and real-world fMRI data. The new slice-based data-analytic framework yields greatly improved signal detection accuracy of fMRI signals.
\end{abstract}

Keywords: fMRI BOLD, detection accuracy, FIR basis functions, statistical modeling, Slice-Based fMRI

Brain function is frequently investigated using the ${ }_{21}$ Blood Oxygen Level Dependent (BOLD) signal in func- 22 tional Magnetic Resonance Imaging (fMRI; Ogawa 23 et al. 1990). Improving the accuracy of methods that 24 detect the BOLD signal is of primary importance in many fMRI research contexts. One recent approach has relied on the implementation of advanced MRI pulsesequences and updated hardware configurations to acquire whole-brain fMRI data with a high temporal resolution (e.g., Chang et al. 2013; Feinberg et al., 2010, Lin et al. 2006, Moeller et al. 2010, van der Zwaag et al., 2006). The higher temporal resolution enables a more precise sampling of the BOLD signal and leads to improved statistical detection and estimation of BOLD signal dynamics in task-based fMRI studies (Chen et al. 2015; Constable \& Spencer, 2001; Dilharreguy et al. 2003, Sahib et al. 2016; Vu et al., 2016; Witt et al. 2016). In addition, a complimentary approach to improve BOLD signal detection has relied on specialized paradigm design and statistical techniques. For exam-

\footnotetext{
*Corresponding author

Email address: njanssen@ull.es (Niels Janssen)
}

ple, past studies have used jittered stimulus presentation with Finite Impulse Response (FIR) modeling to yield higher temporal resolution BOLD signals (e.g., Josephs et al. 1997: Lindquist et al. 2009: Maccotta et al. 2001, Miezin et al., 2000, Price et al., 1999, Serences, 2004, Toni et al. 1999). Here we attempted to further improve these latter data-analytic methods of BOLD signal detection by focusing on two specific issues that hamper the accuracy of BOLD signal extraction: 1) the volumecreation method, and 2) the statistical method.

The first reason why BOLD signal detection in the current fMRI data-analytical framework may be suboptimal is due to the specific method of volume creation. Volume creation refers to the way in which individually acquired brain slices are inserted into whole-brain volumes. A peculiar aspect of fMRI data acquisition is that instead of sampling the entire brain at once, spatially separate brain slices that cover the entire brain are sampled at different moments in time (Cohen \& Weisskoff 1991; Moeller et al., 2010). The current standard practice to create whole-brain volumes from such individually acquired brain slices is to simply time-shift spatially adjacent slices into whole-brain volumes (see

February 7, 2018 
Figure 1 and Appendix 1 for a formal treatment). Given typical whole-brain fMRI sampling parameters of 1 to 3 seconds, this means that time-varying signals sampled from spatially adjacent brain locations may be temporally shifted. Several studies have shown that such data yield BOLD signals that are detected with poor accuracy (Calhoun et al., 2000, Henson et al., 1999, Parker et al., 2017; Sladky et al. 2011). Consequently, these studies also show that signal detection can be improved by a procedure called Slice-Time Correction (STC). STC attempts to alleviate the temporal distortions by either interpolating signals between timepoints (Calhoun et al., 2000, Henson et al., 1999, Sladky et al., 2011), or by first low-pass filtering and then re-aligning signals in time (Parker et al. 2017). However, while these studies demonstrate that STC enhances BOLD signal extraction, it is also clear that STC is only required because of the temporal distortions introduced by the specific method of volume creation. It therefore remains to be seen whether signal extraction can be further improved by alternative methods of volume creation that crucially do not introduce such temporal distortions and hence do not require STC.

A second reason why BOLD signal extraction may be suboptimal is because of the statistical method of signal extraction. Specifically, within the current data-analytical framework, BOLD signal extraction is performed using so-called FIR basis functions (e.g., Josephs et al., 1997; Lindquist et al., 2009; Maccotta et al., 2001, Miezin et al., 2000, Ollinger et al., 2001, Price et al. 1999; Serences, 2004; Toni et al., 1999). The FIR basis functions represent parameters in a General Linear Model (GLM) that each capture a particular point in the progression of the BOLD signal generated by the presentation of stimuli in an imaging run. Formally, within this framework, for a given set of stimuli $S$, the design matrix $X$ with $m$ volumes (rows) and $n$ basis functions (columns) is represented by:

$$
X_{i j}= \begin{cases}1, & \text { if } j=i-\left(S_{p}-1\right) \\ 0, & \text { otherwise }\end{cases}
$$

where $S_{p}$ ranges over all possible volume-based stimulus onsets. The number of basis functions is typically determined by the ratio between the desired epoch length and the repetition time TR and represents the temporal resolution of the extracted signal. Additional basis functions and appropriate jittering of stimuli can be used to increase the temporal resolution (e.g., Josephs et al., 1997; Toni et al., 1999). Given the design matrix $X$ determined above, modeling of fMRI timeseries data $Y$ for a given voxel is performed using the standard GLM function:

$$
Y=X \beta_{0 . . n}+e,
$$

where each $\beta_{j}$ is a value that indexes the strength of the BOLD signal at a particular time point since the presentation of the stimulus. Importantly, given the design of matrix $X$, note that the number of datapoints to go into the estimation of each $\beta_{j}$ value is equal to the number of stimuli in the imaging run (i.e., the number of $1 \mathrm{~s}$ in each column of $X$ ). Approximate values for the $\beta_{j}$ s in this set of linear equations is typically obtained by the least-squares solution:

$$
\beta_{0 . . n}=\left(X^{T} X\right)^{-1} X^{T} Y .
$$

Obtaining an associated t-value with each beta coefficient first involves calculating the mean square error of this model:

$$
\sigma^{2}=\frac{(Y-X b)(Y-X b)^{T}}{n-m},
$$

where the numerator term $Y-X b$ refers to the difference between the obtained and fitted data (i.e., the residuals), and $n-m$ to the available degrees of freedom. Next, the variance associated with each estimated beta-coefficient is given by:

$$
\operatorname{var}\left(\beta_{1 . . n}\right)=\sigma^{2}\left(X^{T} X\right)^{-1},
$$

where the standard error for a given $\beta_{j}$ is obtained by taking the square root of the diagonal values in this matrix. The final t-value can then simply be calculated as the ratio between a given $\beta_{j}$ and its standard error. An appropriate ordering of the beta coefficients or t-values will then result in the statistically extracted BOLD signal.

There are at least three main problems with this FIR based approach that may hamper optimal detection of the BOLD signal. First, parameter estimation in the FIR modeling approach is optimal only if the stimulus induced BOLD signal is stationary across the imaging run (Donnet et al. 2006). Under such conditions, a given $\beta_{j}$ corresponding to a particular timepoint in the BOLD signal progression is estimated from data that contains a minimal amount of distortion in time, and the estimates will therefore be optimal. However, previous studies have observed attentional and top-down influences on the trial-by-trial variability in BOLD signal onset and shape across an imaging run (e.g., Donnet et al., 2006, Duann et al., 2002, Grill-Spector et al., 2006), and therefore undermine the assumption of stationarity. The data from these studies raise the question of whether alternative methods exist that are better suited to address 
the trial-by-trial variability in BOLD signal onset and ${ }_{139}$ shape.

Second, a particular statistical limitation of the GLM ${ }_{141}$ is that it precludes the modeling of random sources 142 of variance such as those due to item variability (e.g., 143 Bedny et al., 2007, Westfall et al., 2016). It is well- 144 known that ignoring sources of variance in the data may 145 introduce biases in parameter estimation. As before, 146 this raises the question of whether BOLD signal de- ${ }_{147}$ tection may be improved by alternative modeling tech- 148 niques in which the aforementioned trial-by-trial vari- 149 ability is brought under statistical control.

Finally, the FIR modeling approach ascribes a rather ${ }_{151}$ counterintuitive meaning to the standard errors associ- 152 ated with the beta $_{j}$ s at each timepoint. Specifically, in ${ }_{153}$ the FIR modeling approach, data from the entire imag- ${ }_{154}$ ing run is used to estimate all the timepoints simultane- 155 ously. This means that the standard error that is asso- 156 ciated with each $\beta_{j}$ corresponding to a particular time- ${ }_{157}$ point is not only determined by the quality of the model 158 fit at that particular timepoint, but by the quality of the 159 model fit at all timepoints (see Equation 5). In other 160 words, the standard error at a particular timepoint does 161 not reflect the quality of data fitting at that particular 162 timepoint alone, but reflects the quality of data fitting 163 at all other timepoints as well. A practical implication 164 of this is that a noise event in the fMRI signal at one 165 particular timepoint will increase the standard error at ${ }_{166}$ all extracted timepoints. Consequently, if BOLD signal 167 extraction relied on t-values, this will affect the accu- 168 racy of BOLD signal extraction at all timepoints, even if 169 the noise event affected only a single timepoint 17 Thus, 170 for these three reasons, the FIR based method of sig- 171 nal extraction may lead to a suboptimal detection of the 172 BOLD signal from fMRI data.

To summarize, within the current framework of fMRI 174 data analysis, BOLD signal extraction is hampered by 175 the specific method of volume creation as well as by the 176 specific method of statistical modeling. Here we pro- 177 posed a new framework for the analysis of fMRI data. 178 This framework incorporates a new method of volume 179 creation, as well as a non-standard technique of statisti- 180 cal signal extraction. The framework places special im- 181 portance on the slice acquisition times, that is the exact ${ }_{182}$ points in time when each slice in the fMRI data stream is 183 acquired. Specifically, in the new method, whole-brain 184 volumes are created out of slices that are all acquired 185 at the same point in time relative to a presented stimu- 186

\footnotetext{
${ }^{1}$ This may suggest that only beta-values should be used. How- ${ }^{188}$ ever, ignoring the standard error introduces new complications in the 189 modeling efforts.
}

lus. This is achieved by presenting stimuli in-phase with the slice acquisition times, and then calculating when each slice was acquired relative to a presented stimulus. These relative acquisition times for each slice can then be used to compose whole-brain volumes in which each slice was acquired at the same moment in time relative to a stimulus. (see Figure 2 and Appendix 1 for a formal treatment). Importantly, this method of wholebrain volume construction does not rely on time-shifting slices as in the standard method. This means that no temporal distortion is introduced in the data and hence, no STC is required.

In addition, in this new fMRI data format, the BOLD signal is extracted using a non-standard Timepoint by Timepoint approach. Although this statistical approach to signal extraction is commonly used in EEG/MEG research (Janssen et al., 2014, Lage-Castellanos et al., 2010; Smith \& Kutas, 2015), it is only rarely applied to fMRI data (but see Cohen et al., 1997; Leung et al., 2000). In the Timepoint by Timepoint approach, the raw, sliced-based fMRI signal is first epoched into time periods where the BOLD response is likely to occur (i.e., stimulus-locked), and then signal intensities from a baseline period (e.g., time points prior to stimulus onset) are compared to signal intensities obtained at later time points in the epoch. Similar to previous studies (e.g., Josephs et al., 1997), because stimuli are presented in-phase with the slice acquisition times, the number of timepoints in an epoch and therefore the maximum temporal resolution with which the BOLD signal can be extracted is determined by $\frac{T R}{\text { num_slices }}$, and may be on the order of tens of milliseconds. Crucially, the Timepoint by Timepoint approach may be less affected by variability in the BOLD signal onset and shape because model coefficients depend on the direct comparison of intensity values between the timepoints in the epoch and the baseline, and leading to more accurate parameter estimation. In addition, parameter estimation in this approach is performed using Linear Mixed Effect (LME) modeling (Bates, 2005, Pinheiro \& Bates, 2000; Westfall et al. 2016). This modern statistical modeling approach permits the inclusion of multiple sources of random variance (see Appendix 2). Finally, because separate models are fitted at each timepoint instead of fitting all timepoints simultaneously, standard errors are less sensitive to potential noise events at other timepoints. Given the central role of slices in this method, we will refer to this framework as Slice-Based fMRI.

The current paper reports on tests that evaluated the accuracy of BOLD signal detection in the new SliceBased method versus the standard FIR based models with STC and without STC. Given that the Slice-Based 
method contains both a new method of volume creation 243 and a different method of statistical signal extraction, 244 a fourth, intermediate model was considered that relied 245 on a standard method of volume creation with STC, but 246 used the Timepoint by Timepoint method of statistical 247 signal extraction. We will refer to this latter model as 248 the Timepoint by Timepoint with STC method. The 249 comparison of these four models allowed for an eval- 250 uation of both the new volume creation method as well 251 as the new Timepoint by Timepoint technique on the 252 accuracy of BOLD signal extraction from fMRI data. 253 Specifically, a contrast of the FIR with STC model with 254 the Timepoint by Timepoint with STC model uses the 255 same volume creation method yet uses a different statis- 256 tical technique and therefore allowed for the evaluation 257 of the new statistical method of signal extraction. In ad- 258 dition, the comparison of the Timepoint by Timepoint 259 with STC and the Slice-Based model uses the same sta- 260 tistical method but relies on different methods of volume creation and therefore allowed for the evaluation of the new volume creation technique.

These four models were evaluated in the context of three simulations and one real-world experiment. The 262 simulations were not designed to examine signal extrac- 263 tion under ideal circumstances, but instead, provided an 264 evaluation of the four models under relatively realistic 265 conditions in an fMRI experiment. In Simulation 1, we 266 examined the impact of trial-by-trial variability in the 267 onset of the BOLD response in consecutive stimulus 268 presentations in an imaging run. In Simulation 2 we ex- 269 amined the impact of trial-by-trial variability in BOLD 270 shape, and in Simulation 3 we examined the impact of 271 a single noise event in the imaging run (a signal inten- 272 sity spike). Each method's performance was examined 273 in the context of a slow event-related imaging run with 274 36 stimuli. The data were sampled from 3 slices con- 275 taining only a single voxel. To examine the impact of 276 increasing the sampling frequency the simulations were 277 repeated with TRs of 3 and 1 second. BOLD signal 278 extraction was performed using t-values. Performance 279 was evaluated in terms of two measures: (i) the Pear- 280 son correlation between the ground-truth signal and the 281 extracted signal, and (ii) the mean absolute difference 282 between the ground-truth signal and the extracted sig- 283 nal. Given the arguments presented above we expected 284 superior performance of the Slice-Based method com- 285 pared to all other methods.

Finally, the four methods were evaluated in the con- 287 text of in-vivo fMRI data collected from 30 participants 288 performing a picture naming task. This task was cho- 289 sen because of its various cognitive components (visual 290 identification, name retrieval from memory, and motor 291 output) which may yield complex BOLD signal dynamics across different areas of the brain. The question was which of the four methods were best suited to detect activity under such conditions. We first evaluated the basic signal detection capabilities of the Slice-Based method by comparing group-level activation maps obtained using this method to the standard GLM and Timepoint by Timepoint methods using Pearson and Dice indices. In addition, we compared BOLD signal extraction using the aforementioned methods from three adjacent slices covering left motor cortex. BOLD signal extraction was compared in terms of four measures: (i) the mean interslice correlation, (ii) the mean number of unique peaks (UP), (iii) the mean Time To Peak (TTP), and (iv) the mean maximum t-value (MAXT). Given the reduced impact of temporal distortions on volume creation and the more sensitive statistical method, we expected better performance for the Slice-Based method.

\section{Methods}

\section{Simulation 1 - variability in BOLD onset}

Simulations were performed in the software $\mathrm{R}$ (v3.4.0) using the neuRosim package (v0.2-12; Welvaert et al., 2011). To simulate an fMRI imaging run, 36 stimuli presented at long $18 \mathrm{~s}$ intervals induced a series of hemodynamic responses that were modeled with a double gamma function with default parameters $(\mathrm{a} 1=6$, $\mathrm{a} 2=12, \mathrm{~b} 1=0.9, \mathrm{~b} 2=0.9, \mathrm{c}=0.35)$. This signal was generated at a very high temporal resolution (accuracy $=0.1$ s). The precise onsets of the stimuli were constructed to be in-phase with the slice acquisition times determined by the fMRI sampling parameters described below. Variability in the onset of the BOLD response was modeled by a stochastic process that for each BOLD response either shifted the onset by $+0.5 \mathrm{~s}$ in time or did not shift onset $(\mathrm{P}=0.5)$. This means that for a given simulation, about 18 out of 36 stimuli yielded a BOLD onset that was $0.5 \mathrm{~s}$ off a (stimulus-induced) stationary onset. If such shifts in onset yield commensurate delays in behavioral response times, then they would yield a standard deviation in response time across all stimuli of around $250 \mathrm{~ms}$. This value is well within the range observed in many behavioral tasks such as picture naming and therefore justifies our choice of realistic parameters for this simulation (e.g., Szekely et al., 2004).

Next, the hemodynamic signal was sampled by three slices in a simple bottom up sequential fashion. Each slice had only a single voxel, meaning that only a single time course was obtained for a given slice. The signal was sampled at two different sampling frequencies. At 
the TR of $3 \mathrm{~s}$ with 3 slices this meant that every slice 344 sampled the signal at a 1 second interval; At the TR of ${ }_{345}$ $1 \mathrm{~s}$ with 3 slices, the signal was sampled at a $0.33 \mathrm{~s}$ in- 346 terval. Thus, each slice sampled the exact same hemo- 347 dynamic response, although as mentioned before, the 348 sequential nature of this serial sampling procedure in- 349 troduces temporal shifts. In the last step of data sam- 350 pling white noise with sigma $=0.15$ was added to the 351 generated time series. Although fMRI data is known 352 to contain other sources of noise (i.e., machine noise, 353 physiological noise), in order to facilitate interpretation 354 it was decided to only add white noise.

Next, three data sets of whole-brain volumes were 356 created from the raw fMRI data. First, a standard vol- 357 ume creation method was used to create a time series 358 of 362 whole-brain volumes in which it was assumed 359 that all three slices within a volume were acquired at 360 the same point in time (see Figure 1). A second data set ${ }_{361}$ was created by applying AFNI's 3dTshift STC function 362 to the first data set. Importantly, signals were aligned 363 to the first slice in the volume meaning that no adjust- 364 ments to the design matrix were required. Interpolation 365 was based on the default Fourier method which is as- 366 sumed to be the most accurate. This therefore yielded 367 a slice time corrected dataset. Finally, the Slice-Based 368 method of volume creation was applied to the raw fMRI 369 data to create a third data set in which all slices within a ${ }_{370}$ volume were acquired at the same moment in time rela- 371 tive to a stimulus (see Figure 2). As mentioned before, 372 this was achieved by combining slices with identical rel- 373 ative acquisition times acquired during the presentation 374 of different stimuli into the same volume. At the TR 375 $=3 \mathrm{~s}$, this epoch had 18 timepoints (i.e., $1 \mathrm{~s}$ tempo- 376 ral resolution), whereas at TR $=1 \mathrm{~s}$ the epoch had 54377 time points (i.e., $0.33 \mathrm{~s}$ temporal resolution). Impor- 378 tantly, these three data sets created by different volume 379 creation methods were always based on the same raw 380 fMRI data.

Statistical extraction of the BOLD signal by the FIR, 382 Timepoint by Timepoint, and Slice-Based methods was 383 performed on these data. For the FIR methods, we con- 384 structed a design matrix with $\frac{\text { epoch_length }}{T R}$ basis functions 385 (e.g., 6 basis functions for an epoch length of $18 \mathrm{~s}$ and 386 a TR of $3 \mathrm{~s}$; See Equation 1). To obtain a temporal res- 387 olution higher than the TR and equal to the resolution 388 obtained using the Slice-Based method, two additional 389 sets of $\frac{\text { epoch_length }}{T R}$ basis functions were added and cor- 390 responded to (jittered) stimulus onsets close to multi- 391 ples of 0.33 and $0.67 *$ TR (e.g., Dale, 1999, Josephs 392 et al., 1997; Price et al., 1999; Toni et al., 1999). This led to a design matrix with a number of parameters that depended on the TR. Specifically, at TR $=3 \mathrm{~s}$ there were 18 parameters in the design matrix, whereas for $\mathrm{TR}=1 \mathrm{~s}$, there were 54 parameters in the design matrix. Note that all basis functions were orthogonal, and that although the number of parameters is high, it remained well below the total number of available datapoints, thereby avoiding overfitting risks. No temporal derivatives were used. This same design matrix was used for the FIR without STC and the FIR with STC methods, where the FIR without STC used the standard dataset for signal extraction, and the FIR with STC used the slice time corrected data set. All statistical modeling was done using the linear modeling $(\mathrm{lm})$ function of R.

For the Timepoint by Timepoint method, epochs were extracted from the standard volume creation dataset with slice-time correction. It was assumed that each volume in the dataset was acquired at the onset of the TR. Next, for each stimulus onset, a set of volumes corresponding to the epoch length were chosen and for each volume in the epoch the relative time since stimulus onset was calculated. BOLD signal extraction took place on the basis of comparing signal intensities at baseline (define as timepoint 0) with those of subsequent timepoints in the epoch. No averaging of data was performed. Model fitting took place using the $\mathrm{R}$ package lme4 (v1.1_13) (Bates, 2005). Specifically, the formula used was lmer (Intensity Time+(1| epoch)), where Time was a fixed-effect factor with two levels (the baseline and the relevant timepoint), and epoch was random-effect variable referring to the item number. Finally, the Slice-Based method used the same signal extraction method as the Timepoint by Timepoint method, except that the volume creation method was slice-based and not volume-based. This difference in volume creation method may lead to more accurate signal extraction in the Slice-Based method for two reasons: First, given that no STC is required, and hence no data is interpolated, extraction of a more veridical signal is expected than in the Timepoint by Timepoint with STC method. Second, given that in the Slice-Based method the onset and offsets of epochs are determined by the precise slice-acquisition times and not by the TRbased volume acquisition times, extracted epochs correspond more closely to actual stimulus onsets and offsets and therefore result in a more precise allocation of datapoints to timepoints in the epoch than in the Timepoint by Timepoint method. This improved alignment may then result in a more accurate extraction of the BOLD signal (see Discussion and Supplementary Materials for further discussion of this point).

Performance of each model was evaluated by the comparison to a ground-truth signal. Because the original signal was specified in different units than the statis- 
tically extracted signal, no direct comparisons were pos- 422 sible. Instead, the ground-truth signal was set to have ${ }_{423}$ a maximum t-value amplitude of 25. This amplitude of the ground-truth signal was found to be sufficiently high ${ }_{424}$ such that the simulations performed with the particular ${ }_{425}$ noise levels did not reach this value. The ground-truth ${ }_{426}$ signal was then calculated with this maximum effect- ${ }_{427}$ size parameter using the double gamma function that formed the basis of the original fMRI data. Importantly, the same ground-truth signal was used across all simulations and was the same for all four evaluated methods. The accuracy of BOLD signal detection was determined using two measures: First, accuracy was determined by the Pearson correlation between the ground-truth signal and the signal at a particular slice. The mean Pearson correlation (denoted $\bar{r}_{1}$ ) was then computed as the mean correlation across all slices. In addition, the accuracy was also determined by the mean absolute distance between the ground truth and the signal at a particular slice:

$$
d=\frac{\sum_{i=1}^{n}\left|a_{i}-b_{i}\right|}{n}
$$

where $n$ is the number of timepoints in the epoch, $a$ is the ground truth signal and $b$ is the extracted BOLD signal at a given slice. The value $\bar{d}$ was then calculated as the mean $d$ value across all slices. The main advantage of this distance measure over a Pearson cor- 445 relation is that the distance measure takes into account ${ }^{446}$ the amplitude of the response and therefore provides ${ }^{447}$ a more precise indication of the degree to which the ${ }^{448}$ extracted BOLD signal approximated the ground-truth ${ }^{449}$ signal. Note that lower $\bar{d}$ values indicate a more closely ${ }^{450}$ extracted signal. In total 100 simulations were per- ${ }^{451}$ formed at each TR.

\section{Simulation 2 - variability in BOLD shape}

In Simulation 2, the impact of variability in the 455 BOLD shape across an imaging run on BOLD signal extraction by the four methods was examined. Vari- ${ }^{456}$ ability in the BOLD shape was modeled by changing 457 the parameter values of the double gamma function that 458 was used to generate the baseline BOLD signal. Specif- 459 ically, for half the stimuli in this simulation experiment, 460 the BOLD response was generated by a double gamma ${ }_{461}$ function with adjusted values $(\mathrm{a} 1=6, \mathrm{a} 2=12, \mathrm{~b} 1=0.7,462$ $\mathrm{b} 2=0.7, \mathrm{c}=0.25$ ), while the other half had default pa- 463 rameter values (see above). Note that the b1 parameter 464 controls the dispersion of the response, the b2 parameter 465 controls the dispersion of the undershoot, and that the $\mathrm{c} 466$ parameter controls the scale of the undershoot. With re- ${ }_{467}$ spect to the default settings in the gamma function, these 468 parameters were reduced to yield a BOLD response that 469 was slightly more narrow. All other aspects of Simulation 2 were identical to Simulation 1.

\section{Simulation 3 - impact of single spike}

In Simulation 3, the impact of a single intensity spike on BOLD signal extraction by the four methods was investigated. This spike was modeled by changing a single intensity value in the fMRI simulated time series of slice 1 at a timepoint that was sampled at the end of an epoch (i.e., during the BOLD undershoot). This particular intensity value at this timepoint was set to 5 times the maximum BOLD signal (i.e., the maximum BOLD signal was 1 , the value was set to 5 ). In other words, the fMRI time series of slice 1 consisted of 362 time points, and the intensity value at a single timepoint that was located at the end of a stimulus induced BOLD signal was set to 5 times the maximum BOLD signal. Intensity values at all other 361 timepoints for slice 1 remained unchanged. Note that such spikes in the signal are a frequent occurrence in fMRI data and are thought to be the result of head motion and the resulting spin-history artifacts (e.g., Friston et al., 1996).

\section{In-vivo data - Picture Naming}

\section{Participants}

Thirty native speakers of Spanish took part in the experiment (20 females, 10 males, mean age 22 yrs). Participants were students at the University of La Laguna, and received course credit or were paid 10 Euro. Twenty-nine participants were right-handed. The study was conducted in compliance with the declaration of Helsinki, and all participants provided informed consent in accordance with the protocol established by the Ethics Commission for Research of the university of La Laguna (Comit de tica de la Investigacin y Bienestar Animal).

\section{Experimental setup and procedure}

Two stimuli were used in the task: First, an image which participants were asked to name aloud, and second, a fixation cross ('+') which indicated rest (see Figure 3 for an overview). Twenty-seven pictures were selected from an image database that contained standardized line-drawings that were normed on various aspects (Szekely et al. . 2004). Only those images were selected that had names that were consistently produced across participants in the norming study (i.e., those with $\& 90 \%$ name-agreement).

Stimuli were presented in a slow event-related design, where a stimulus was presented for $0.5 \mathrm{~s}$ followed by an ISI blank screen for $12 \mathrm{~s}$ plus an additional jitter period. 
The duration of the jitter period was randomly chosen 519 without replacement from a uniform distribution of $36{ }_{520}$ times from 0 to $1855 \mathrm{~ms}$ in steps of $53 \mathrm{~ms}$. This method ${ }_{521}$ of stimuli presentation resulted in the optimal jittering ${ }_{522}$ of stimuli for the Slice-Based method (see Figure 2 for ${ }_{523}$ further details). Stimulus presentation was directly syn- ${ }_{524}$ chronized with the MRI machine.

The Experiment involved three consecutive runs. In ${ }^{526}$ each run, 36 stimuli were presented, of which half were ${ }^{527}$ pictures and half were rest (i.e, fixation cross). In each 528 run, nine different pictures were randomly selected and ${ }_{529}$ which were presented twice. Different pictures were se- 530 lected for each run, and all twenty-seven pictures were 531 presented in the experiment. For each run, the order 532 of the stimuli was fully randomized on a by-participant 533 basis. Stimulus presentation was controlled by Neurobs 534 Presentations (v14). Participants in the scanner viewed 535 the stimuli with MRI compatible goggles made by Vi- 536 suaStim. These goggles provided an image resolution ${ }_{537}$ of 800 by 600 pixels at $60 \mathrm{~Hz}$.

\section{MRI acquisition parameters}

MR-images were acquired using a 3T Signa Excite scanner (General Electric, Milwaukee, WI, USA) using a standard transmit/receive 8 channel gradient head coil. Head movement was strenuously avoided by fixating each participant's head with spongepads inside the coil. T2*-weighted images were obtained using standard Gradient Echo, Echo Planar Imaging (EPI) sequences.

Each run started with 10 dummy volumes that al- ${ }_{50}$ lowed for steady-state tissue magnetization. Each volume contained 36 slices that were acquired top-down, axially and interleaved. Slice thickness was $3.7 \mathrm{~mm}$ with $0.3 \mathrm{~mm}$ gap. The FOV was $256 \times 256 \mathrm{~mm}$, matrix size $64 \times 64$, resulting in $4 \times 4 \times 4 \mathrm{~mm}$ isometric voxels. ${ }_{555}$ TR was $1908 \mathrm{~ms}$, echo time (TE) $21.6 \mathrm{~ms}$, and the flip 556 angle $75^{\circ}$. This unusual TR was chosen because it was ${ }_{557}$ the fastest TR possible in the context of the other pa- ${ }_{558}$ rameter settings and therefore would generate the maximum amount of data. In addition, 1908 is a multiple of 36 and this simplifies determining the slice acquisition times and stimulus presentation times. In each run 255 volumes were collected and lasted 8 minutes and 6 seconds.

Separate high resolution T1-weighted images were 565 acquired using the 3D FSPGR sequence: TI/TR/TE: 566 $650 / 6.8 / 1.4 \mathrm{~ms}$, flip angle $=12^{\circ}, 196$ slices, slice thick- ${ }_{567}$ ness $1 \mathrm{~mm}$, matrix $256 \times 256$, voxel size $=1 \times 1 \times 1568$ $\mathrm{mm}$.

\section{Pre-processing}

Only minimal data pre-processing was applied: Low frequency drifts were removed using a high pass filter at $0.01 \mathrm{~Hz}$ (Smith et al., 2004), and the data were motion corrected using FSL MCLFLIRT (Jenkinson et al., 2002). Note that in the context of the Slice-Based method, motion correction poses a certain challenge. This is because the Slice-Based method not only assumes that a given voxel samples the same physical brain area throughout the imaging run (as in all fMRI methods), but also assumes that this voxel is sampled at regular well-known time intervals. Motion correction may lead to the translation of a physical brain area across slices and therefore impact the time interval at which this brain area was sampled. To address this issue we implemented a method for motion correction that allowed for standard spatial motion correction and provided an additional temporal correction that updates the time intervals of the physical areas underlying the voxels that were sampled (see Janssen et al. (submitted) for further details). However, it should be pointed out that our data set did not include a sufficiently large amount of motion to accurately verify the efficacy of this motion correction method. Finally, a second data set was created that was slice-time corrected using the AFNI $3 d T$ shift using the first slice as a reference and the standard Fourier interpolation method. The STC function was applied before the motion correction. Spatial smoothing was not used in any of the data sets.

\section{Comparison of activation maps}

The activation map for the standard method was obtained using the GLM method implemented in FSL Feat (Jenkinson et al., 2012) and used the STC dataset described above. Precise picture naming onsets were extracted from the participant-specific Presentation logfiles. The rest periods were not explicitly modeled (Pernet. 2014). The expected HRF was modeled as a double gamma function with default parameters, pre-whitening was applied, and the temporal derivative was included in the GLM model. The analysis only included the first run of each participant. A final group level map was generated by performing a one-sample t-test on the standard space transformed beta-coefficient maps of each participant using FSL randomise (Winkler et al. 2014).

The activation maps for the Timepoint by Timepoint with STC and Slice-Based method were constructed by comparing signal intensities at a baseline timepoint between one TRs before and one TR after stimulus presentation to signal intensities at timepoints between one and 5 TRs following stimulus presentation (i.e., capturing the peak of the BOLD signal). As in the simulation 
data, signal intensities were modeled using LME with ${ }_{621}$ the lme4 package in R. Signal intensities were modeled 622 as a function of a fixed effect variable Timepoint (signal ${ }_{623}$ intensities at baseline versus timepoint 1). In addition, 624 the variations that arise due to items were brought into 625 the model by including a random intercept for Item (i.e., 626 the formula was Imer (Intensity Time+(1| Item)). 627 This model therefore provides for each participant a single activation map that indicates how signal intensity increased or decreased at timepoint 1 relative to baseline. The group level maps for the Timepoint by Timepoint and Slice-Based methods were generated in the exact same way as for the standard method. Note that as we ${ }^{629}$ mentioned earlier, the Timepoint by Timepoint method is volume-based and therefore may introduce uncertain- 630 ties in the signal extraction compared to the Slice-Based 631 method. Note also that the same transformation matri- ${ }^{632}$ ces to normalize each participant's scanner space image 633 to standard space were used between the three methods. ${ }^{634}$

\section{BOLD signal extraction from left motor cortex}

BOLD signals were extracted from three adjacent ${ }^{637}$ slices in the left motor cortex that all showed strong 638 involvement in the task. To identify the active voxels ${ }^{639}$ on adjacent slices in left motor cortex we first created 640 a mask of each participant's left precentral gyrus using ${ }^{641}$ the lateralized Harvard-Oxford probabilistic atlas (De- ${ }^{642}$ sikan et al. 2006). Any voxels included in the mask 643 on the medial surface of the left hemisphere were re- 644 moved as those regions are unlikely to be involved in 645 primary motor cortex control of speech. Next, the voxel 646 with the maximum t-value in the masked GLM signal 647 detection map (see above) was identified for each par- 648 ticipant, which corresponds to the voxel with the max- 649 imum t-value in the left motor cortex. This resulted in 650 three times series from adjacent slices in left motor cor- 651 tex that were strongly involved in the task. This set of 652 three time series for every participant formed the input 653 to the four techniques.

Specifically, the BOLD signals extracted from these 655 three slices in left motor cortex were examined with the 656 FIR without STC, FIR with STC, Timepoint by Time- 657 point with STC, and Slice-Based methods. The extrac- 658 tion was performed exactly as described above using 659 the simulated data. Note no temporal derivates were ${ }_{660}$ included in the model. We examined the mean inter- 661 slice correlation, the mean UP, the mean TTP, and the 662 mean MAXT in the BOLD signal across the three slices 663 for all participants. The TTP was calculated in the stan- 664 dard way as the mean timepoint at which the extracted 665 BOLD signal at each slice reached its maximum value. 666 The UP indicated the number of unique peaks found for 667 each slice and was calculated by counting the number of unique TTPs found across slices. The Maximum t-value was the maximum t-value across all slices. We extracted the BOLD signal in the thee slices in left motor cortex at two temporal resolutions, the TR (1908 ms), and $\mathrm{TR} / 2$ (954 ms). We performed statistical comparisons of these values on a by-participant basis.

\section{Results}

\section{Simulation - variability in BOLD onset}

A graphical presentation of a single representative result from this simulation experiment is presented in Figure 4. A visual impression of this result suggested that the Slice-Based method yielded the BOLD signals in closest correspondence with the ground-truth signal (dashed line). Given the high value of the mean correlation with the ground-truth signal $\left(\bar{r}_{1}\right)$ for all methods (suggesting ceiling effects), the analyses were focused on the mean absolute difference between the groundtruth signal and the mean extracted signal across all slices $(\bar{d})$. An overview of the means and statistics of the $\bar{d}$ value for each method across all simulations in presented in Figure 7

For the simulation on the variability in the onset of the BOLD signal, the analyses revealed that for $\mathrm{TR}=3$, the lowest $\bar{d}$ values were observed for the Slice-Based method. Specifically, the Slice-Based method yielded a lower $\bar{d}$ value compared to FIR without STC $(\mathrm{F}(1,198)$ $=3555.5, \mathrm{p} ; 0.0001)$, FIR with STC $(\mathrm{F}(1,198)=$ 1589.8, p ; 0.0001), and Timepoint by Timepoint with STC methods $(\mathrm{F}(1,198)=8.7, \mathrm{p} ; 0.004)$. In addition, the Timepoint by Timepoint with STC differed from the FIR without STC $(\mathrm{F}(1,198)=4170.3, \mathrm{p} ; 0.0001)$, and the FIR with STC methods $(\mathrm{F}(1,198)=1761.6, \mathrm{p}$ $i$ 0.0001). Finally, the FIR with STC differed from the FIR without STC method $(\mathrm{F}(1,198)=747.1, \mathrm{p}$; $0.0001)$.

Likewise, for TR $=1$, the Slice-Based method again had the lowest $\bar{d}$ values. In particular, the Slice-Based method had lower $\bar{d}$ values than FIR without STC $\left(\mathrm{F}(1,198)=350.4, \mathrm{p}_{i}\right.$ 0.0001), FIR with STC $(\mathrm{F}(1,198)$ $=67.4, \mathrm{p} ; 0.0001)$, and Timepoint by Timepoint with STC methods $(F(1,198)=5.3, p ; 0.03)$. And as before, the Timepoint by Timepoint with STC differed from the FIR without STC $(F(1,198)=258.0, p ; 0.0001)$, and the FIR with STC methods $(\mathrm{F}(1,198)=27.9, \mathrm{p} ; 0.0001)$. Finally, the FIR with STC differed from the FIR without STC method $(F(1,198)=482.6, p ; 0.0001)$. 


\section{Simulation 2 - variability in BOLD dispersion}

As with the previous simulation, a visual impression of Figure 5 suggested that the Slice-Based method was also superior under conditions of variability in peak BOLD signal dispersion. The statistical analyses revealed that for $\mathrm{TR}=3$, the Slice-Based method yielded a lower $\bar{d}$ value compared to FIR without STC $(\mathrm{F}(1,198)$ $\left.=2158.3, p_{i} 0.0001\right)$, FIR with $\operatorname{STC}(F(1,198)=269.8$, $\mathrm{p} ; 0.0001)$, and Timepoint by Timepoint with STC methods $(F(1,198)=35.1, p ; 0.0001)$. In addition, the Timepoint by Timepoint with STC differed from the FIR without STC $(\mathrm{F}(1,198)=1780.8, \mathrm{p} ; 0.0001)$, and the FIR with STC methods $(\mathrm{F}(1,198)=94.3$, p $i$ 0.0001). Finally, the FIR with STC differed from the FIR without STC method $(\mathrm{F}(1,198)=3480.4, \mathrm{p}$; $0.0001)$.

Likewise, for TR $=1$, the Slice-Based method also had lower $\bar{d}$ values than FIR without STC $(\mathrm{F}(1,198)=$ $163.8, \mathrm{p} ; 0.0001)$, FIR with STC $(\mathrm{F}(1,198)=74.2, \mathrm{p}$; $0.0001)$, and Timepoint by Timepoint with STC methods $(F(1,198)=26.5, p$; 0.0001). And as before, the Timepoint by Timepoint with STC differed from the FIR without STC $(\mathrm{F}(1,198)=43.7, \mathrm{p} ; 0.0001)$, and marginally from the FIR with STC methods $(\mathrm{F}(1,198)$ $=4.1, \mathrm{p}=0.05)$. Finally, the FIR with STC differed from the fir without STC method $(F(1,198)=189.7, p$ $0.0001)$.

\section{Simulation 3 - impact of single spike}

In line with previous simulations, Figure 6 suggested that the Slice-Based method extracted a more accurate BOLD signal when a single spike was present in the data for a single voxel. The statistical analyses revealed that regarding the $\bar{d}$ value, for TR $=3$, the Slice-Based 749 method differed from FIR without STC $\left(\mathrm{F}(1,198)={ }_{750}\right.$ $3941.4, p ; 0.0001)$, FIR with STC $(\mathrm{F}(1,198)=595.2,751$ $p_{i}$ 0.0001), but not from Timepoint by Timepoint with 752 STC methods $(\mathrm{F}(1,198)=2.7, \mathrm{p}=0.10)$. In addition, 753 the Timepoint by Timepoint with STC differed from 754 the FIR without STC $(F(1,198)=3629.7, p ; 0.0001), 755$ and the FIR with STC methods $(\mathrm{F}(1,198)=489.3$, p 756 i 0.0001). Finally, the FIR with STC differed from 757 the FIR without STC method $\left(\mathrm{F}(1,198)=7673.5, \mathrm{p} ;{ }_{758}\right.$ 0.0001).

Likewise, for TR $=1$, the Slice-Based method also 760 had lower $\bar{d}$ values than FIR without STC $(\mathrm{F}(1,198)=761$ 210.2, p ; 0.0001), FIR with STC $(\mathrm{F}(1,198)=30.2, \mathrm{p} 762$ i 0.0001), but not Timepoint by Timepoint with STC 763 methods $(\mathrm{F}(1,198)=0.22, \mathrm{p}=0.63)$. As previously, 764 the Timepoint by Timepoint with STC differed from the 765 FIR without STC $(F(1,198)=121.3, p ; 0.0001)$, and 766 from the FIR with STC methods $\left(\mathrm{F}(1,198)=14.5, \mathrm{p}_{i}\right.$ 0.0002). Finally, the FIR with STC differed from the fir without STC method $(F(1,198)=883.4, p ; 0.0001)$.

\section{In-vivo Results}

\section{Comparison of activation maps}

Figure 8 presents the comparison of the whole-brain group analysis of overt picture naming using the standard GLM with STC obtained with FSL Feat (panel A), a map obtained using the Timepoint by Timepoint with STC method (panel B), and that of the Slice-Based method (panel $\mathrm{C}$ ). Inspection of the difference maps (Figure 8, panels D, E, F, and G), revealed that the SliceBased method yielded substantially higher t-values in some areas of the brain such as the medial frontal cortex compared to the other two methods (see Figure 8 panels D and E). Quantitative comparisons confirmed these visual impressions. The Pearson's correlation coefficient between the non-thresholded and vectorized versions of the Slice-Based and GLM maps was high at 0.95 ( $\mathrm{p}$; 0.001 ) and between the Slice-Based and Timepoint by Timepoint map was 0.94 ( $\mathrm{p} ; .001$ ), suggesting comparable activation patterns. However, the Dice index (see black line Figure 9), revealed that at higher t-value thresholds, the similarity between the Slice-Based and GLM map (Figure 9 panel A) and between the SliceBased and Timepoint by Timepoint map (Figure 9 panel B) decreased. Further examination revealed that this decrease was due to the presence of more active voxels in the Slice-Based map at higher t-value thresholds (see gray line Figure 9), indicating improved signal detection for the Slice-Based method.

\section{BOLD signal extraction from left motor cortex}

Figure 10 provides an overview of the extracted BOLD signals for the four methods from the three adjacent slices in left motor cortex for three representative participants from a single imaging run with 18 stimuli at the TR temporal resolution of $1.908 \mathrm{~s}$. Figure 11 relied on the same data but BOLD signals were extracted at twice the temporal resolution $(0.954 \mathrm{~s})$. Overall the visual impression is that the Slice-Based method extracted the BOLD signal with increased t-values. A graphical overview of the means and statistical differences for each method can be seen in Figure 12 Statistical analyses confirmed these visual impressions. Specifically, for the $\mathrm{TR}=1.908 \mathrm{~s}$, the interslice correlation was highest for the FIR with STC (mean = 0.76) and differed from the FIR without STC $(0.69 ; \mathrm{t}(29)=4.2, \mathrm{p} ; 0.0003)$ and Slice-Based methods $(0.73 ; \mathrm{t}(29)=3.1, \mathrm{p} ; 0.004)$. For the TR $=0.954 \mathrm{~s}$, Slice-Based and FIR with STC had 
comparable interslice correlations. In addition, the in- 817 terslice correlation of the FIR with STC model differed 818 from the Timepoint by Timepoint $\left(0.62 ; \mathrm{t}(29)=2.4,{ }_{819}\right.$ $\mathrm{p} ; 0.03)$ and FIR without STC $(0.59 ; \mathrm{t}(29)=4.7, \mathrm{p} ; 820$ 0.0001 ) methods.

Regarding the mean unique number of peaks across 822 each slice, for TR $=0.954 \mathrm{~s}$, the Slice-Based method ${ }_{823}$ had the highest number of unique peaks (2.6), and dif- 824 fered from the Timepoint by Timepoint STC $(2.3 ; \mathrm{t}(29) 825$ $=2.1, \mathrm{p} ; 0.05)$, the FIR with STC $(2.1 ; \mathrm{t}(29)=3.3, \mathrm{p} 826$ $i 0.003)$, and the FIR without STC $\left(2.3 ; \mathrm{t}(29)=2.6, \mathrm{p} ;{ }_{827}\right.$ 0.02 ) methods.

For the mean time to peak, at TR $=0.954 \mathrm{~s}$, the Time- 829 point by Timepoint model had the earliest time to peak 830 (4.0), and differed from the FIR without STC method 831 $(4.4 ; t(29)=2.2, p ; 0.04)$.

For the mean maximum $\mathrm{t}$-value observed across all ${ }_{833}$ slices, at TR $=1.908 \mathrm{~s}$, the Slice-Based method had the ${ }_{834}$ higest mean maximum t-value (9.2), and differed from 835 the Timepoint by Timepoint with STC $(8.6 ; \mathrm{t}(29)=2.7,836$ $\left.\mathrm{p}_{i} 0.02\right)$, the FIR with STC $(8.4 ; \mathrm{t}(29)=2.2, \mathrm{p} ; 0.04), 837$ and FIR without STC $(7.9 ; \mathrm{t}(29)=3.7, \mathrm{p} ; 0.001)$ meth- 838 ods. In addition, the FIR without STC had a lower max- 839 imum t-value than the FIR with STC $(\mathrm{t}(29)=4.4, \mathrm{p} ; 840$ 0.0002). Similarly, at TR $=0.954 \mathrm{~s}$, the Slice-Based 841 method again had the higest maximum t-value (8.7), 842 and differed from the Timepoint by Timepoint with STC 843 $(7.8 ; \mathrm{t}(29)=3.1, \mathrm{p} ; 0.005)$, the FIR with STC $(6.7 ; 844$ $\mathrm{t}(29)=5.6, \mathrm{p} ; 0.0001)$, and without STC $(6.4 ; \mathrm{t}(29) 845$ $=6.4, \mathrm{p} ; 0.0001)$ methods. In addition, the Timepoint 846 by Timepoint method differed from both FIR with STC ${ }_{847}$ $(\mathrm{t}(29)=3.4, \mathrm{p} ; 0.003)$ and without STC $\left(\mathrm{t}(29)=4.2, \mathrm{p}{ }_{848}\right.$ $i$ 0.0003), and the FIR with STC differed from the FIR 849 without STC (t(29) = 3.5, p; 0.002) methods.

Finally, the above results give the impression that the 851 Slice-Based method was more robust against increased 852 in temporal resolution compared to the FIR based meth- ${ }_{853}$ ods. Further analyses confirmed this impression: For 854 the FIR with STC method, the statistical difference in 855 maximum $\mathrm{t}$-value between TR and TR/2 was larger 856 $(t(59)=-9.11, \mathrm{p} ; 0.0001)$ than for the Slice-Based ${ }_{857}$ method $(\mathrm{t}(59)=-2.55, \mathrm{p} ; 0.02)$, suggesting that the 858 Slice-Based method indeed provides better BOLD sig- 859 nal extraction accuracy under conditions of increased 860 temporal resolution than the FIR based methods.

\section{Discussion}

The current study evaluated four methods that dif- 865 fered in how the BOLD signal can be extracted from ${ }_{866}$ fMRI data. These four methods differed in how volumes 867 were created from individually acquired brain slices, 868 and in how the statistical extraction of the BOLD signal takes place. Specifically, whereas the FIR without STC, FIR with STC, and Timepoint by Timepoint with STC methods all relied on the same standard volume creation technique (see Figure 1), the Slice-Based method relied on a new volume creation technique that does not require STC (see Figure 2). In addition, statistical extraction of the BOLD signal relied on the standard FIR with GLM approach (i.e., the FIR without and with STC methods), or a non-standard Timepoint by Timepoint approach using LME modeling (i.e., the Timepoint by Timepoint with STC, and Slice-Based methods). Results from three simulation experiments revealed that the best performance was observed for the Slice-Based method (mean increased accuracy in terms of $\bar{d}$ of SliceBased vs FIR with STC for TR 3 was $32 \%$, for TR 1 it was $13 \%$ ). Similarly, analyses of real-world data revealed that the Slice-Based method yielded dramatically improved signal detection in group level maps (at a t-value threshold of 8 , the Slice-Based map contained about 3 times more active voxels compared to the standard GLM map, and 8 times more active voxels compared to the Timepoint by Timepoint map), as well as higher maximum t-values of the extracted BOLD signal in the motor cortex (mean increased accuracy in terms of maximum t-value of Slice-Based vs FIR with STC for TR resolution was $9 \%$, for TR/2 it was $24 \%$ ). Overall, the Slice-Based method significantly improved BOLD signal extraction accuracy compared to the standard methods.

Before discussing the main implications, two aspects of these data deserve further scrutiny. First, in the realworld data, the FIR with STC method yielded a higher inter-slice correlation $\left(\bar{r}_{2}\right)$ at the TR resolution and a lower number of unique peaks (UP) at the TR/2 resolution than the Slice-Based method (see Figure 12]. This may suggest that the FIR with STC method yielded more coherency in the BOLD signals extracted from different slices than the Slice-Based method. However, note that this increase in similarity between signals on adjacent slices was directly caused by the STC function that effectively smoothed the data, which led to more similar signals on adjacent slices (e.g., Parker et al., 2017; Sladky et al. 2011). However, it is not obvious that these more similar signals reflect the veridical underlying signal. Indeed, although the simulation studies revealed that STC increased the accuracy with which the ground-truth signal was extracted, these studies also revealed that this accuracy of signal extraction in the FIR with STC method was relatively poor when compared to the accuracy of the Slice-Based method (see Figure 77. Thus, whereas the FIR with STC method will pro- 
duce signals that appear similar across adjacent slices, 921 this does not necessarily imply more accurate signal 922 detection, and the simulation results indicated BOLD ${ }_{923}$ signals were extracted more accurately with the Slice- 924 Based method.

Another aspect of the data that deserves further men- 926 tion is that whereas signal detection accuracy greatly 927 improved due to the Timepoint by Timepoint statisti- 928 cal method, additional improvements due to the slice- 929 based method of volume creation were seemingly more 930 modest (comparing FIR with STC, Timepoint by Time- 931 point with STC to Slice-Based methods, see Figure 7D). 932 Given that the Slice-Based technique comprises both the ${ }_{933}$ Timepoint by Timepoint technique and the new volume 934 creation method, one possible conclusion from these re- 935 sults is therefore that the main contribution of the Slice- 936 Based framework lies primarily in the statistical model- 937 ing technique and not in the new method of volume cre- 938 ation. However, this conclusion would be premature for 939 the following three reasons: First, note that in the sim- 940 ulation experiments, although improvements between 941 the Timepoint by Timepoint and Slice-Based methods 942 were modest, they were statistically significant (Figure 943 7D). In addition, in the real-world data, there were rela- 944 tively large differences between the Timepoint by Time- 945 point and the Slice-Based methods in terms of the max- 946 imum extracted t-value (especially at $954 \mathrm{~ms}$ temporal ${ }_{947}$ resolution, see Figure 12D). Finally, as mentioned ear- 948 lier, epoching of data is more precise in the Slice-Based 949 framework, leading to more accurate allocation of dat- 950 apoints to timepoints in the Slice-Based method com- 951 pared to the Timepoint by Timepoint method. Addi- 952 tional simulations presented in the Supplementary Ma- 953 terials (see Figure S2 and S3) further highlight condi- 954 tions in which the Timepoint by Timepoint with STC 955 method yielded inaccurate signal detection (and may ex- 956 plain the differences observed in Figure 12D). Overall, 957 these observations suggest that the new method of Slice- 958 Based volume creation confers additional advantages of 959 BOLD signal detection over and above the advantages 960 already accrued by the Timepoint by Timepoint method 961 of statistical signal extraction.

How did the new volume creation technique improve 963 BOLD signal extraction accuracy? There are two major 964 differences between the new and the standard method 965 of volume creation. First, as mentioned in the Introduc- 966 tion, in the new method, whole-brain volumes contain 967 slices with signals that are temporally aligned, mean- 968 ing that there are no within volume temporal distor- 969 tions of signals across adjacent slices. By contrast, in 970 the standard method these signals are not temporally 971 aligned, meaning that there are within volume tempo- 972 ral distortions of signals across slices (cf., Figures 1 and 2). The important implication of the presence of temporally aligned signals across slices within consecutive volumes is that the STC procedure is no longer required. Removing the STC procedure from the fMRI processing pipeline means that less data transformations are required, and this means that in the new method a more accurate and veridical BOLD signal can be extracted. Second, the new volume creation method leads to a reorganization of the fMRI data that fundamentally differs from the standard method of volume creation. Specifically, the new method reorganizes the data into stimulus-locked epochs where each datapoint in this epoch is labeled with timing information that is determined by the exact slice acquisition times (on the order of tens of milliseconds). By contrast, in the standard method, datapoints are labeled with timepoints determined by the TR (on the order of hundreds to thousands of milliseconds). This means that in the Slice-Based volume creation technique, timing information is much more precise. In our data, the availability of this highly precise timing information enabled statistical modeling approaches that improved the accuracy of BOLD signal extraction. Thus, the new volume creation technique improved the accuracy of BOLD signal extraction because it removes the need for STC and it includes more precise timing information in the data.

Besides the new volume creation technique, the current study also introduced a new method of statistical BOLD signal extraction. Specifically, the modeling approach used in the current study relied on a Timepoint by Timepoint technique. While the method of comparing timepoints in an epoch to a baseline timepoint has been used in previous fMRI studies (e.g., Leung et al., 2000), the specific implementation that uses LME modeling proposed here is new. This modeling approach improved BOLD signal extraction over the standard FIR based GLM approach in three ways. First, in the Timepoint by Timepoint technique, model coefficients are estimated by comparing signal intensities at all timepoints in an epoch to a baseline timepoint (e.g., data collected prior to stimulus onset). The direct comparison of signal intensities between a baseline and a timepoint means that more data is available for coefficient estimation compared to a no baseline FIR model (as used here), avoids problems associated with subtracting model coefficients in FIR models that use a baseline, and avoids the need for explicit baseline (rest) periods in the experimental design. This leads to more stable model estimates in BOLD signal extraction. In addition, the specific statistical modeling technique used in the current study was LME modeling implemented in 
R (Bates, 2005; Pinheiro \& Bates, 2000). A major ad- 1025 vantage of LME modeling over the standard GLM con- 1026 cerns the use of more complex random effect structures 1027 (Westfall et al., 2016). For example, the current study 1028 included random-intercepts for the effect of item in the ${ }_{1029}$ statistical model. The inclusion of known sources of ${ }_{1030}$ variance in the model leads to more stable model esti- 1031 mates, and improved signal extraction. Finally, because ${ }_{1032}$ in the Timepoint by Timepoint approach separate mod- ${ }_{1033}$ els are fitted at each timepoint, the standard errors are ${ }_{1034}$ less sensitive to sudden noise events at other timepoints 1035 in the epoch (Friston et al., 1996), and hence, lead to 1036 more stable estimates. In short, the Timepoint by Time- 1037 point approach for statistical signal extraction improved ${ }_{1038}$ the accuracy of BOLD signal extraction over the stan- ${ }_{1039}$ dard GLM based methods.

As mentioned in the Introduction, current advances 1041 in MRI pulse sequence design and hardware coils have ${ }_{1042}$ enabled the acquisition of fMRI data at very short 1043 TRs. For example, several studies have shown that with 1044 Simultaneous Multi-Slice acquisition methods (SMS), 1045 whole-brain volumes can be acquired at TRs of 5001046 ms and less (Chen et al., 2015, Feinberg \& Setsom- 1047 pop, 2013, Vu et al. 2016). Interestingly, although 1048 reductions in TR are generally associated with lower 1049 SNR, studies have demonstrated that the TR reductions 1050 yielded increased statistical power, suggesting that the ${ }_{1051}$ additional data offset the reductions in SNR (Constable ${ }_{1052}$ \& Spencer, 2001; Sahib et al., 2016). The Slice-Based ${ }_{1053}$ method proposed here may be combined with these ex- 1054 isting SMS techniques to further improve BOLD sig- 1055 nal detection. In practical terms, this combination is 1056 straightforward since all that the Slice-Based method re- 1057 quires is a list of the specific timepoints at which each ${ }_{1058}$ slice was acquired. Although the impact of temporal 1059 distortions produced by time-shifting slices is expected 1060 to be reduced with short TRs in SMS techniques (Sahib ${ }_{1061}$ et al., 2016), further minimizing the impact of such dis- 1062 tortions can be achieved with the Slice-Based method. 1063 In addition, the Slice-Based method has the advantage ${ }_{1064}$ of not requiring state-of-the-art MRI pulse sequences or 1065 hardware, but can extract BOLD signals with high tem- 1066 poral resolution and accuracy at long TRs with good ${ }_{1067}$ SNR using run-of-the-mill pulse sequences. In short, 1068 the Slice-Based method could improve signal detection 1069 for existing cutting-edge techniques, as well as provide 1070 a method for BOLD signal extraction with high tem- 1071 poral resolution and accuracy that is not restricted by 1072 specific hardware configurations.

The current study has several limitations. First, as 1074 explained in the Method section, although head mo- 1075 tion is considered a problem for all fMRI techniques, 1076 it poses special challenges to Slice-Based fMRI. Although the results presented here relied on the standard procedures implemented in FSL to alleviate head motion artefacts (i.e., MCFlirt), they were accompanied by specialized Slice-Based fMRI procedures developed to alleviate any temporal distortions induced by head motion (see Janssen et al., submitted for details). Although these specialized Slice-Based motion correction procedures improved signal detection (Janssen et al., submitted), further validation of these procedures is required in dedicated future studies. Second, the current study relied on real-world fMRI data collected from a picture naming task. We are currently planning studies that use different tasks in which expectations about BOLD signal dynamics are more easily verified, such as, for example, inducing delayed BOLD responses between left and right motor cortex (e.g., Menon et al., 1998, Miezin et al., 2000). Third, although the Slice-Based technique works with both slow event-related and blocked designs, it currently does not work for fast event-related designs. Note, however, that fast event-related designs pose challenges to all linear modeling approaches (e.g., Vazquez \& Noll, 1998). Finally, the Slice-Based framework requires more statistical tests than the standard FIR based approaches. In the Slice-Based framework, a statistical model is fitted to each voxel in the brain at each timepoint, and therefore raises issues about multiple comparisons. In our data we dealt with this issue by using high t-value thresholds. In the future, this issue may be addressed by implementing 4D cluster correction techniques such as those available for EEG and MEG data (Guthrie \& Buchwald, 1991, Maris \& Oostenveld, 2007). To facilitate further development on all these issues an example data set and tutorial script for analyzing an fMRI data set within the SliceBased framework is available at https://github. com/iamnielsjanssen/slice-based. In brief, we think that despite these limitations the Slice-Based technique is a feasible alternative for the analysis of fMRI data.

To conclude, current methods for extracting BOLD signals rely on volumes constructed by time-shifting slices, STC, and FIR-based statistical modeling techniques (Josephs et al. 1997). Here we show that such an approach leads to a relatively poor detection of the true underlying BOLD signal. By contrast, the SliceBased framework that was proposed here relies on a fundamentally different method of volume construction, does not require STC, and uses non-standard Timepoint by Timepoint modeling for signal extraction. The results from both simulated and real-world data showed that this new fMRI data-analytic method led to im- 
proved BOLD signal detection accuracy. Although the

1078 Slice-Based framework places more strict constraints on

1079 paradigm design, the new framework is a relatively sim-

1080 ple data-analytic method that does not require special-

1081 ized MRI hardware, is more robust against trial-by-trial

1082 variability in BOLD onset and shape, is more robust

1083 against sudden noise events, allows for an easy appli-

1084 cation of modern LME modeling techniques, and can

1085 be applied to a wide variety of fMRI research contexts

1086 that rely on both event-related and blocked designs. U1-

1087 timately we think that further improvements in BOLD

1088 signal detection will come from a combination of the

1089 Slice-Based framework with newer hardware oriented

1090 techniques. 
Bates, D. M. (2005). Fitting linear mixed models in r. R News, 5, 1156 27-30.

Bedny, M., Aguirre, G. K., \& Thompson-Schill, S. L. (2007). Item 1158 analysis in functional magnetic resonance imaging. Neuroimage, 1159 35, 1093-1102.

Calhoun, V., Golay, X., \& Pearlson, G. (2000). Improved fmri slice 1161 timing correction: interpolation errors and wrap around effects. In 1162 Proceedings, ISMRM, 9th annual meeting, Denver (p. 810). 1163

Chang, W.-T., Nummenmaa, A., Witzel, T., Ahveninen, J., Huang, S., 1164 Tsai, K. W.-K., Chu, Y.-H., Polimeni, J. R., Belliveau, J. W., \& Lin, 1165 F.-H. (2013). Whole-head rapid fmri acquisition using echo-shifted 1166 magnetic resonance inverse imaging. Neuroimage, 78, 325-338. 1167

Chen, L., Vu, A., Xu, J., Moeller, S., Ugurbil, K., Yacoub, E., \& Fein- 1168 berg, D. (2015). Evaluation of highly accelerated simultaneous 1169 multi-slice epi for fmri. Neuroimage, 104, 452-459.

Cohen, J. D., Perlstein, W. M., Braver, T. S., Nystrom, L. E., Noll, 1171 D. C., Jonides, J., \& Smith, E. E. (1997). Temporal dynamics of 1172 brain activation during a working memory task. Nature, 386, 604. 1173

Cohen, M. S., \& Weisskoff, R. M. (1991). Ultra-fast imaging. Mag- 1174 netic resonance imaging, 9, 1-37.

Constable, R. T., \& Spencer, D. D. (2001). Repetition time in echo 1176 planar functional mri. Magnetic resonance in medicine, 46, 748- 1177 755.

Dale, A. M. (1999). Optimal experimental design for event-related 1179 fmri. Human brain mapping, 8, 109-114.

Desikan, R. S., Ségonne, F., Fischl, B., Quinn, B. T., Dickerson, B. C., 1181 Blacker, D., Buckner, R. L., Dale, A. M., Maguire, R. P., Hyman, 1182 B. T. et al. (2006). An automated labeling system for subdividing 1183 the human cerebral cortex on mri scans into gyral based regions of 1184 interest. Neuroimage, 31, 968-980.

Dilharreguy, B., Jones, R. A., \& Moonen, C. T. (2003). Influence of 1186 fmri data sampling on the temporal characterization of the hemo- 1187 dynamic response. Neuroimage, 19, 1820-1828.

Donnet, S., Lavielle, M., \& Poline, J.-B. (2006). Are fmri event- 1189 related response constant in time? a model selection answer. $\mathrm{Neu}-1190$ roimage, 31, 1169-1176.

Duann, J.-R., Jung, T.-P., Kuo, W.-J., Yeh, T.-C., Makeig, S., Hsieh, 1192 J.-C., \& Sejnowski, T. J. (2002). Single-trial variability in event- 1193 related bold signals. Neuroimage, 15, 823-835.

Efron, B., \& Morris, C. (1977). Stein's paradox in statistics. Scientific 1195 American, 236, 119-127.

Feinberg, D. A., Moeller, S., Smith, S. M., Auerbach, E., Ramanna, 1197 S., Glasser, M. F., Miller, K. L., Ugurbil, K., \& Yacoub, E. (2010). 1198 Multiplexed echo planar imaging for sub-second whole brain fmri 1199 and fast diffusion imaging. PloS one, 5, e15710.

Feinberg, D. A., \& Setsompop, K. (2013). Ultra-fast mri of the human 1201 brain with simultaneous multi-slice imaging. Journal of magnetic 1202 resonance, 229, 90-100.

Friston, K., Williams, S., Howard, R., Frackowiak, R., \& Turner, R. 1204 (1996). Movement-related effects in fmri time-series. Magnetic 1205 resonance in medicine, 35, 346-355.

Grill-Spector, K., Henson, R., \& Martin, A. (2006). Repetition and 1207 the brain: neural models of stimulus-specific effects. Trends in 1208 cognitive sciences, 10, 14-23.

Guthrie, D., \& Buchwald, J. S. (1991). Significance testing of differ- 1210 ence potentials. Psychophysiology, 28, 240-244.

Henson, R., Buechel, C., Josephs, O., \& Friston, K. (1999). The slice- 1212 timing problem in event-related fmri. NeuroImage, 9, 125.

Janssen, N., Hernández-Cabrera, J. A., \& Ezama Foronda, L. (submit- 1214 ted). Data on motion correction in slice-based functional magnetic 1215 resonance imaging. Data in Brief, Janssen, N., Hernández-Cabrera, J. A., van der Meij, M., \& Barber, 1217 H. A. (2014). Tracking the time course of competition during word 1218 production: Evidence for a post-retrieval mechanism of conflict 1219 resolution. Cerebral Cortex, (p. bhu092).
Jenkinson, M., Bannister, P., Brady, M., \& Smith, S. (2002). Improved optimization for the robust and accurate linear registration and motion correction of brain images. Neuroimage, 17, 825-841. Jenkinson, M., Beckmann, C. F., Behrens, T. E., Woolrich, M. W., \& Smith, S. M. (2012). Fsl. Neuroimage, 62, 782-790.

Josephs, O., Turner, R., \& Friston, K. (1997). Event-related fmri. Human brain mapping, 5, 243-248.

Lage-Castellanos, A., Martínez-Montes, E., Hernández-Cabrera, J. A., \& Galán, L. (2010). False discovery rate and permutation test: an evaluation in erp data analysis. Statistics in medicine, 29, 63-74.

Leung, H.-C., Skudlarski, P., Gatenby, J. C., Peterson, B. S., \& Gore, J. C. (2000). An event-related functional mri study of the stroop color word interference task. Cerebral cortex, 10, 552-560.

Lin, F.-H., Wald, L. L., Ahlfors, S. P., Hämäläinen, M. S., Kwong, K. K., \& Belliveau, J. W. (2006). Dynamic magnetic resonance inverse imaging of human brain function. Magnetic resonance in medicine, 56, 787-802.

Lindquist, M. A., Loh, J. M., Atlas, L. Y., \& Wager, T. D. (2009). Modeling the hemodynamic response function in fmri: efficiency, bias and mis-modeling. Neuroimage, 45, S187-S198.

Maccotta, L., Zacks, J. M., \& Buckner, R. L. (2001). Rapid selfpaced event-related functional mri: feasibility and implications of stimulus-versus response-locked timing. Neuroimage, 14, 11051121.

Maris, E., \& Oostenveld, R. (2007). Nonparametric statistical testing of eeg-and meg-data. Journal of neuroscience methods, 164, 177190.

Menon, R. S., Luknowsky, D. C., \& Gati, J. S. (1998). Mental chronometry using latency-resolved functional mri. Proceedings of the National Academy of Sciences, 95, 10902-10907.

Miezin, F. M., Maccotta, L., Ollinger, J., Petersen, S., \& Buckner, R. (2000). Characterizing the hemodynamic response: effects of presentation rate, sampling procedure, and the possibility of ordering brain activity based on relative timing. Neuroimage, 11, 735-759.

Moeller, S., Yacoub, E., Olman, C. A., Auerbach, E., Strupp, J., Harel, N., \& Uğurbil, K. (2010). Multiband multislice ge-epi at 7 tesla, with 16-fold acceleration using partial parallel imaging with application to high spatial and temporal whole-brain fmri. Magnetic Resonance in Medicine, 63, 1144-1153.

Ogawa, S., Lee, T.-M., Kay, A. R., \& Tank, D. W. (1990). Brain magnetic resonance imaging with contrast dependent on blood oxygenation. Proceedings of the National Academy of Sciences, 87, 9868-9872

Ollinger, J., Shulman, G. L., \& Corbetta, M. (2001). Separating processes within a trial in event-related functional mri: I. the method. Neuroimage, 13, 210-217.

Parker, D., Liu, X., \& Razlighi, Q. R. (2017). Optimal slice timing correction and its interaction with fmri parameters and artifacts. Medical image analysis, 35, 434-445.

Pernet, C. R. (2014). Misconceptions in the use of the general linear model applied to functional mri: a tutorial for junior neuroimagers. Frontiers in neuroscience, 8 .

Pinheiro, J. C., \& Bates, D. M. (2000). Mixed effects models in S and $S$-PLUS. Springer.

Price, C. J., Veltman, D., Ashburner, J., Josephs, O., \& Friston, K. (1999). The critical relationship between the timing of stimulus presentation and data acquisition in blocked designs with fmri. NeuroImage, 10, 36-44.

Sahib, A., Mathiak, K., Erb, M., Elshahabi, A., Klamer, S., Scheffler, K., Focke, N., \& Ethofer, T. (2016). Effect of temporal resolution and serial autocorrelations in event-related functional mri. Magnetic resonance in medicine, 76, 1805.

Serences, J. T. (2004). A comparison of methods for characterizing the event-related bold timeseries in rapid fmri. Neuroimage, 21, 
1690-1700.

1222 Sladky, R., Friston, K. J., Tröstl, J., Cunnington, R., Moser, E., \& Windischberger, C. (2011). Slice-timing effects and their correction in functional mri. Neuroimage, 58, 588-594.

1225 Smith, N. J., \& Kutas, M. (2015). Regression-based estimation of erp waveforms: Ii. nonlinear effects, overlap correction, and practical considerations. Psychophysiology, 52, 169-181.

228 Smith, S. M., Jenkinson, M., Woolrich, M. W., Beckmann, C. F., Behrens, T. E., Johansen-Berg, H., Bannister, P. R., De Luca, M., Drobnjak, I., Flitney, D. E. et al. (2004). Advances in functional and structural $\mathrm{mr}$ image analysis and implementation as fsl. $\mathrm{Neu}$ roimage, 23, S208-S219.

Szekely, A., Jacobsen, T., D’Amico, S., Devescovi, A., Andonova, E., Herron, D., Lu, C. C., Pechmann, T., Pléh, C., Wicha, N. et al (2004). A new on-line resource for psycholinguistic studies. Journal of memory and language, 51, 247-250.

Toni, I., Schluter, N. D., Josephs, O., Friston, K., \& Passingham, R. E. (1999). Signal-, set-and movement-related activity in the human brain: an event-related fmri study. Cerebral cortex, 9, 35-49.

Vazquez, A. L., \& Noll, D. C. (1998). Nonlinear aspects of the bold response in functional mri. Neuroimage, 7, 108-118.

Vu, A. T., Phillips, J. S., Kay, K., Phillips, M. E., Johnson, M. R., Shinkareva, S. V., Tubridy, S., Millin, R., Grossman, M., Gureckis, T. et al. (2016). Using precise word timing information improves decoding accuracy in a multiband-accelerated multimodal reading experiment. Cognitive Neuropsychology, 33, 265-275.

Welvaert, M., Durnez, J., Moerkerke, B., Verdoolaege, G., \& Rosseel, Y. (2011). neurosim: An r package for generating fmri data. Journal of Statistical Software, 44, 1-18.

Westfall, J., Nichols, T., \& Yarkoni, T. (2016). Fixing the stimulusas-fixed-effect fallacy in task fmri. bioRxiv, (p. 077131).

Winkler, A. M., Ridgway, G. R., Webster, M. A., Smith, S. M., \& Nichols, T. E. (2014). Permutation inference for the general linear model. Neuroimage, 92, 381-397.

Witt, S. T., Warntjes, M., \& Engström, M. (2016). Increased fmri sensitivity at equal data burden using averaged shifted echo acquisition. Frontiers in Neuroscience, 10.

van der Zwaag, W., Francis, S., \& Bowtell, R. (2006). Improved echo volumar imaging (evi) for functional mri. Magnetic resonance in medicine, 56, 1320-1327. 


\section{Appendix 1}

Formally, fMRI data $D$ can be represented as a set of $m$ slices $S$ that are repeatedly sampled $n$ times:

$$
D=\left[S_{1,1}, \ldots, S_{m, n}\right]
$$

where each $S$ is itself a two dimensional matrix of acquired fMRI signal intensities (not shown here). This data matrix of slices $D$ is accompanied by a similar size $m \times n$ matrix of slice acquisition times $D T$.

$$
D T=\left[t_{1,1}, \ldots, t_{m, n}\right]
$$

Under the assumption of a standard sequential slice acquisition scheme, each specific time point $t(a, b)$ in this matrix can be determined by the following function:

$$
t(a, b)=\frac{T R}{m} \times(a+(b-1) \times m),
$$

where $v$ ranges from 1 to $n$.

In the Slice-Based method, volume-creation requires a set $P$ of $m$ stimuli $\left[p_{1}, \ldots, p_{m}\right]$, whose corresponding ${ }_{1267}$ stimulus presentation times $P T$ coincide precisely with ${ }_{1268}$ the slice acquisition times determined by equation 8

$$
P T=\left[p t_{1, a}, \ldots, p t_{m, y}\right]=\left[t_{1, a}, \ldots, t_{m, y}\right],
$$

where $j$ correspond to the slice acquired during stimulus presentation, and $k$ to the slice acquired at the end of an epoch. The corresponding set of slice acquisition times for an epoch is:

$$
E T_{j}=\left[t_{j, a}, \ldots, t_{k, d}\right]
$$

where each specific time point in this set is determined by Equation 8 . Next, for each given epoch $E_{j}$ we compute the relative time difference $R E T_{j}$ between the exact presentation time of the stimulus $p t(j, a)$ and each time point in the epoch:

$$
R E T_{j}=\left[t_{j, a}-p t_{j, a}, \ldots, t_{k, d}-p t_{j, a}\right]=\left[r t_{j, a}, \ldots, r t_{k, d}\right] .
$$

Following this step, we create a single epoch $L$ with $r$ whole-brain volumes

$$
L=\left[V_{1}, \ldots, V_{r}\right]
$$

where $r$ is determined by the ratio between the epoch length $\Delta t$ and the slice sampling frequency $\frac{T R}{m}$. The corresponding vector of volume acquisition times $L T$ is determined by

$$
L T=\left[l t_{1}, \ldots, l t_{r}\right],
$$

where each $l t$ is determined by the function:

$$
l t(v)=\frac{T R}{m} \times v .
$$

Each volume in the epoch $L$ contains slices that are acquired at the same time point relative to the onset of the stimulus. This is achieved by combining slices from different epochs $E_{1}, \ldots, E_{m}$ on the basis of their $R E T$ values. Specifically slices $1, \ldots, m$ can be combined into a whole-brain volume if their corresponding relative times $r t$ match. For a given volume:

$V_{e}=\left[S_{1, a}, S_{2, d}, \ldots, S_{m, y}\right] \Longleftrightarrow r t_{1, a}=r t_{2, d}=\ldots=r t_{m, y}$.

This then leads to an epoch of whole-brain volumes that do not contain any temporal distortions, and where volumes are available at a temporal resolution equal to the sampling frequency. Finally, note that binning across timepoints may be used to improve the SNR. In this case, the temporal resolution is determined by the ratio between the epoch length and the number of bins.

Next, we create $m$ epochs $E_{1}, \ldots, E_{m}$ corresponding to each stimulus presentation. Each epoch has length $\Delta t$. A given epoch $E_{j}$ then contains raw fMRI signal intensities as defined as the set of slices:

$$
E_{j}=\left[S_{j, a}, \ldots, S_{k, d}\right]
$$




\section{Appendix 2}

The particular statistical modeling technique that is used in the Slice-Based framework is called Linear Mixed Effect modeling (LME; Pinheiro \& Bates 2000). LME modeling is best seen as an extension of the GLM, meaning that both techniques are multiple linear regression techniques. However, LME modeling offers a number of advantages over the GLM technique. First, the LME technique is a multilevel technique which can handle complex random effect structures. This means that, for example, fMRI data can be modeled while taking into account random variability between participants and items, instead of just between participants as is currently the standard. Second, the LME technique can handle unbalanced data sets. This means experimental conditions can have missing values, and model estimation will still work. Finally, the LME parameter estimation takes into account a statistical phenomenon known as shrinkage (Efron \& Morris, 1977). This means that parameter estimates are adjusted in the context of what the model knows about the other estimates, thereby preventing overfitting of the model. Overall, these features improve model estimation and reduce the probability of biased parameter estimates.

One way to formally present a LME model is as follows:

$$
\begin{aligned}
& Y=X \beta+Z \gamma+\epsilon, \\
& \gamma \sim N_{r}(0, G), \\
& \epsilon \sim N_{N}(0, R),
\end{aligned}
$$

where $Y$ is a $N \times 1$ vector of fMRI signal intensities at a particular voxel $p$, at a particular time point $t, X$ is the design matrix of size $(N \times(1+k)), \beta$ are the estimates of the fixed effects $((1+k) \times 1), Z$ is the design matrix of the random effect predictors with $g$ clusterings (e.g., items) and $r$ random effects $(N \times(r \times g)), \gamma$ the random effect estimates $(r \times g) \times 1$, and $\epsilon$ the residual errors. The $\gamma$ parameter is assumed to be a random variable chosen from a normal distribution with a mean of zero and a covariance matrix $G$, while the residual error $\epsilon$ is assumed to be normally distributed with mean of zero and covariance matrix $R$. The unknown model parameters for $\beta$, $G$, and $R$ can be estimated using Maximum Likelihood or Restricted Maximum Likelihood methods. The parameter $\gamma$ is not a parameter of the model but its values are simply derived once the other parameters have been discovered. 


\section{Acknowledgements}

1314 See https://github.com/iamnielsjanssen/ 1315 slice-based for a full analysis script using the 1316 Slice-Based method. This work was supported by The 1317 Spanish Ministry of Economy and Competitiveness 1318 (RYC2011-08433 and PSI2013-46334 to NJ). The 1319 authors would like to thank Cristin Modroo (SEGAI, ${ }_{1320}$ Universidad de La Laguna) and Jos Mara Prez Gon1321 zlez for technical support, and Sara Duque Gonzlez,

1322 Rebeca de Luis Sosa, and Alba Rodrguez Gonzlez ${ }_{1323}$ for help with data collection. Correspondence and 1324 requests for materials should be addressed to $\mathrm{NJ}$ 1325 (njanssen@ull.es). 
$1326 \quad$ Figures 


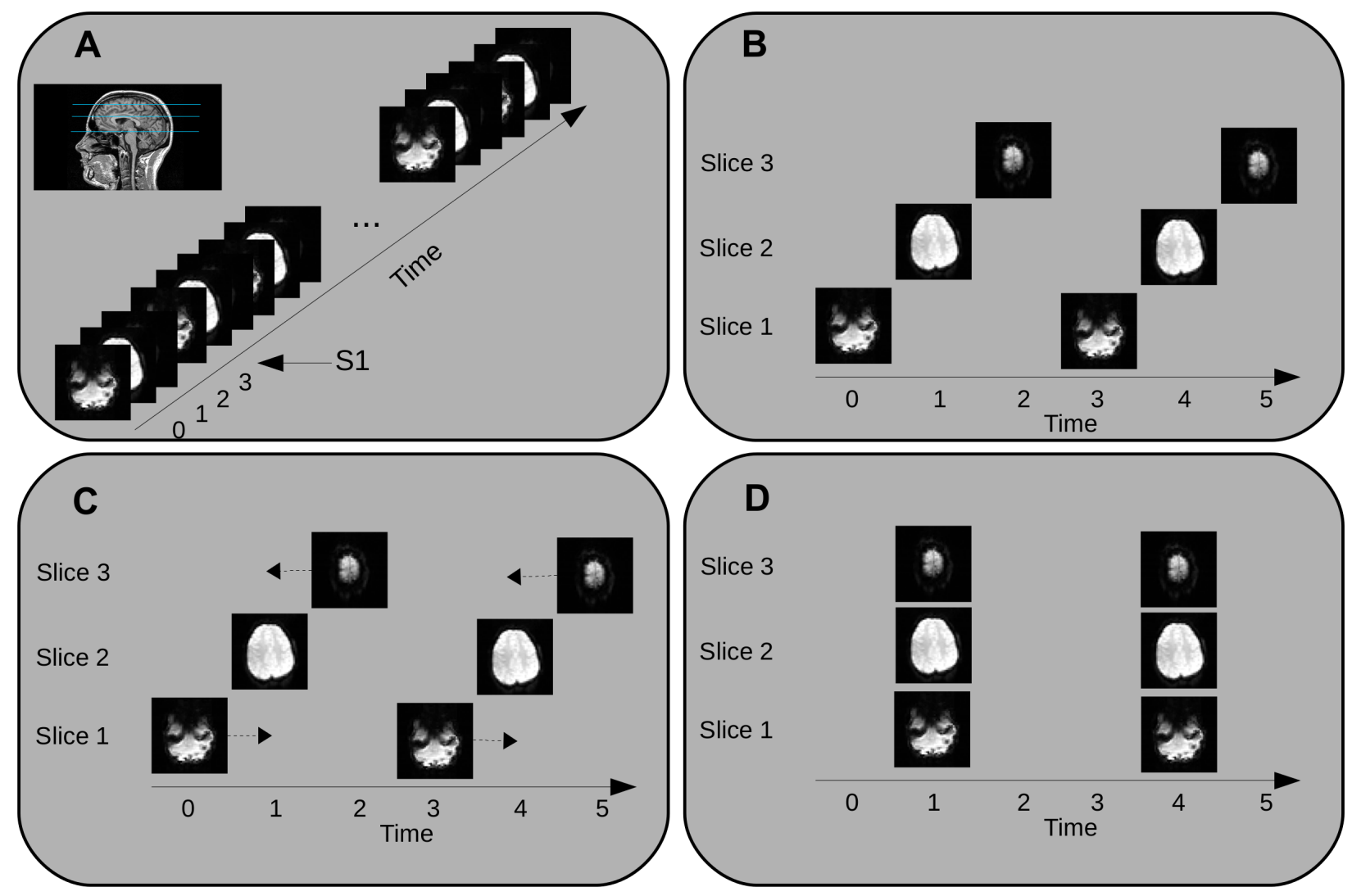

Figure 1: Current standard method for creating whole-brain volumes from raw fMRI data. Panel A shows an imaging run where a set of three slices are sequentially sampled at well defined points in time. Panel B reveals the same data, reorganized to illustrate that at no sampled time point information from the whole-brain is available, requiring data transformation. Panel $\mathrm{C}$ shows the standard solution, where slices are time-shifted to new positions in time (arrows indicate shift direction), using the middle slice as an arbitrary reference. Panel D shows the final transformed data, where whole-brain volumes are available every TR. Note how the final volumes contain slices acquired at different points in time, and how time points where data was sampled are no longer used. 


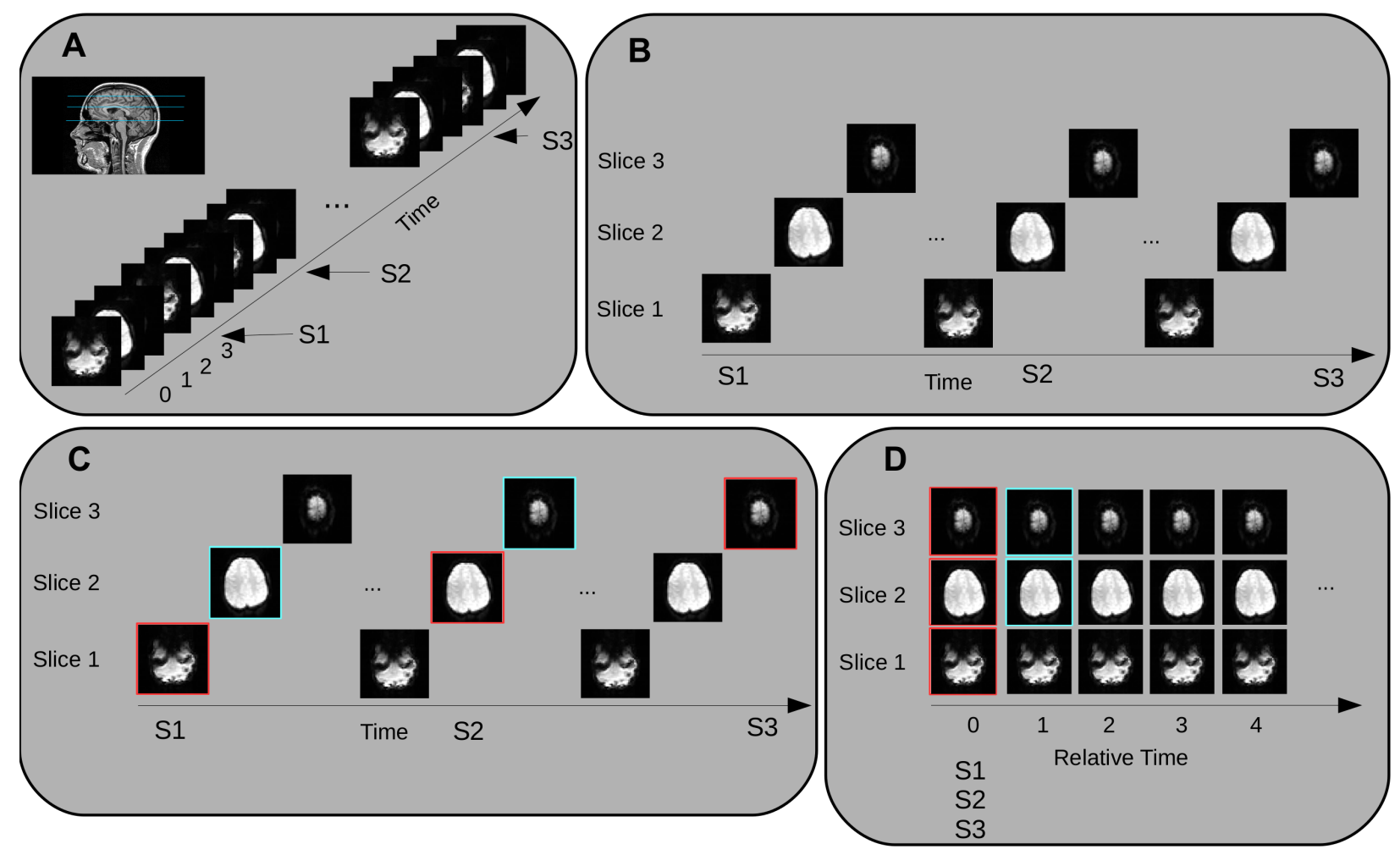

Figure 2: Slice-based method for creating whole-brain volumes from raw fMRI data. Panel A shows an imaging run where again three slices are sampled sequentially. Three stimuli S1, S2, and S3 of the same experimental class are presented during the run. Panel B shows that these stimuli are presented in-phase with slice acquisitions: S1 is presented in-phase with acquisition of slice 1, S2 with slice 2, and S3 with slice 3. Panel C shows how whole-brain volumes are created. Slices acquired at the same point in time relative to the onset of a stimulus can be combined (e.g., those highlighted in red and magenta). Panel D shows the final transformed data, where whole-brain volumes are available that only contain slices that are acquired at the same moment in time relative to a presented stimulus, and where whole-brain volumes are available at the sampling frequency (here TR/3). 


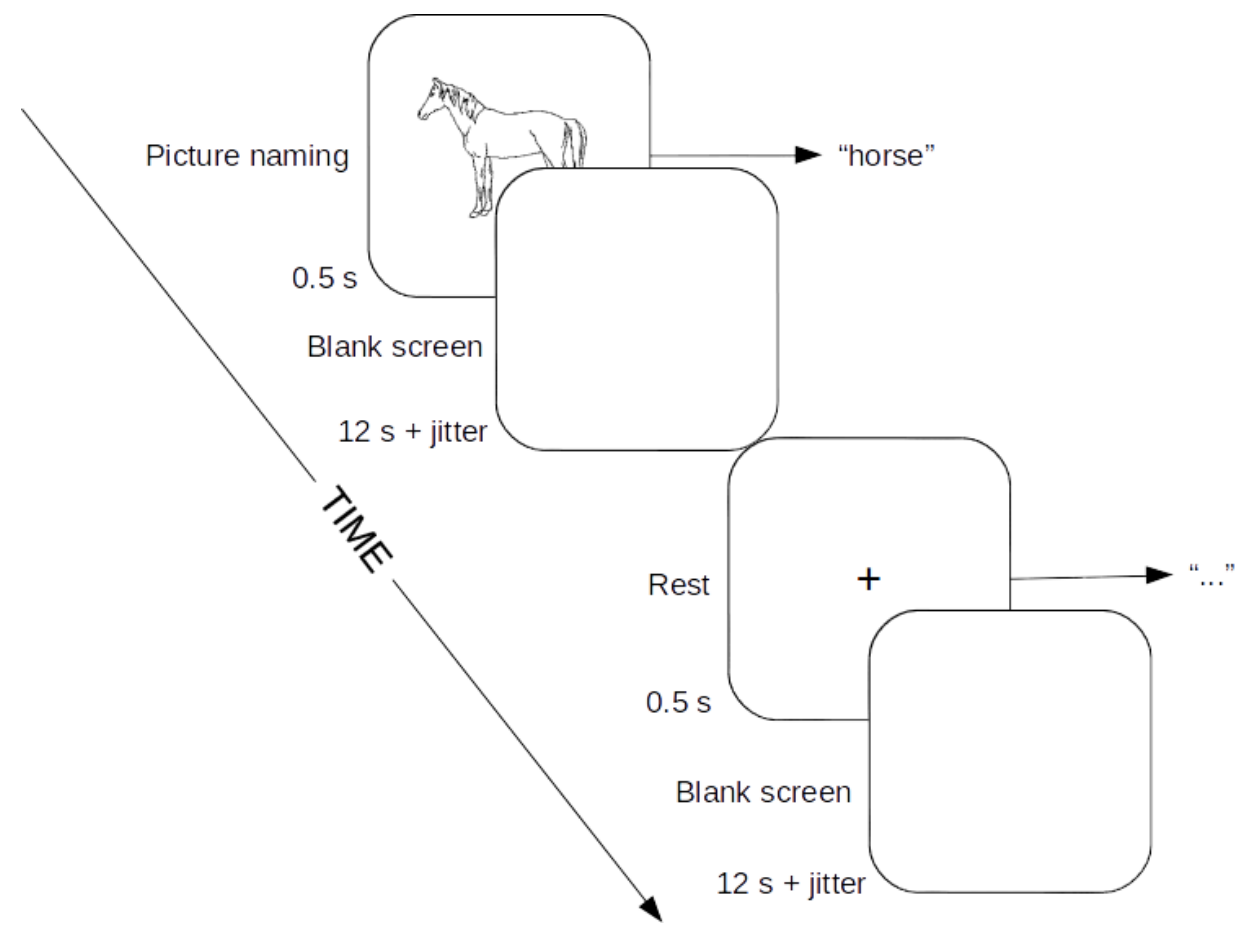

Figure 3: Temporal structure of the picture naming task used in the experiment. Stimuli consisted of either a picture or a fixation point that was presented for $0.5 \mathrm{~s}$. Each stimulus presentation was followed by a blank screen that lasted for $12 \mathrm{~s}$ plus an additional jitter period. The jitter period was randomly selected without replacement from a uniform distribution of times that coincided with the slice acquisition times and ranged from 0 to $1855 \mathrm{~ms}$ in steps of $53 \mathrm{~ms}$ (see text for further details). Participants were instructed to name aloud presented pictures and remain quiet (i.e., rest) for presented fixation points. The order of stimuli presentation was fully randomized, and was different for every participant. 

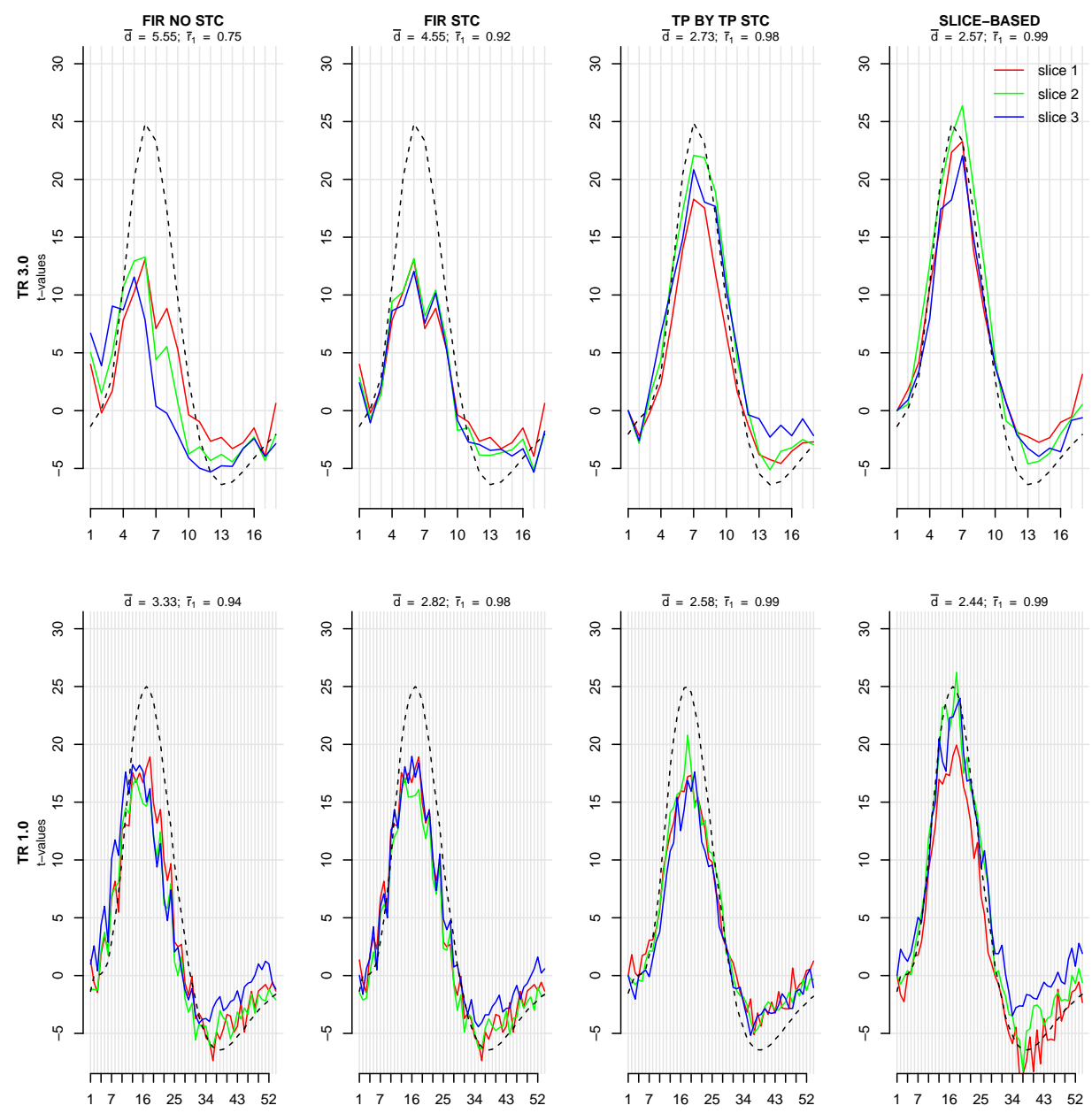

Figure 4: Differences in the detection accuracy of the BOLD signal due to trial-by-trail variability in the onset of the BOLD signal in a simulated fMRI experiment. Each column in the figure represents a different method (first column = FIR without STC; second $=$ FIR with STC; third = Timepoint by Timepoint with STC; fourth $=$ Slice-Based), and each row represents a different TR $($ TR $=3$ top row; TR $=1$, bottom row). BOLD response variability was modeled by randomly delaying its onset by 0.5 seconds for half the stimuli. In all simulations, white noise was modeled with $\sigma=0.15$. Shown are the extracted signals from a single representative simulation. Figure titles list the mean absolute difference between the ground-truth signal and the extracted signals across slices $(\bar{d})$, and the mean correlation between the ground-truth signal and the signal from each slice $\left(\bar{r}_{1}\right)$. Note the overall high $\bar{r}_{1}$ values, and that the lowest $\bar{d}$ values are found in the Slice-Based method. 

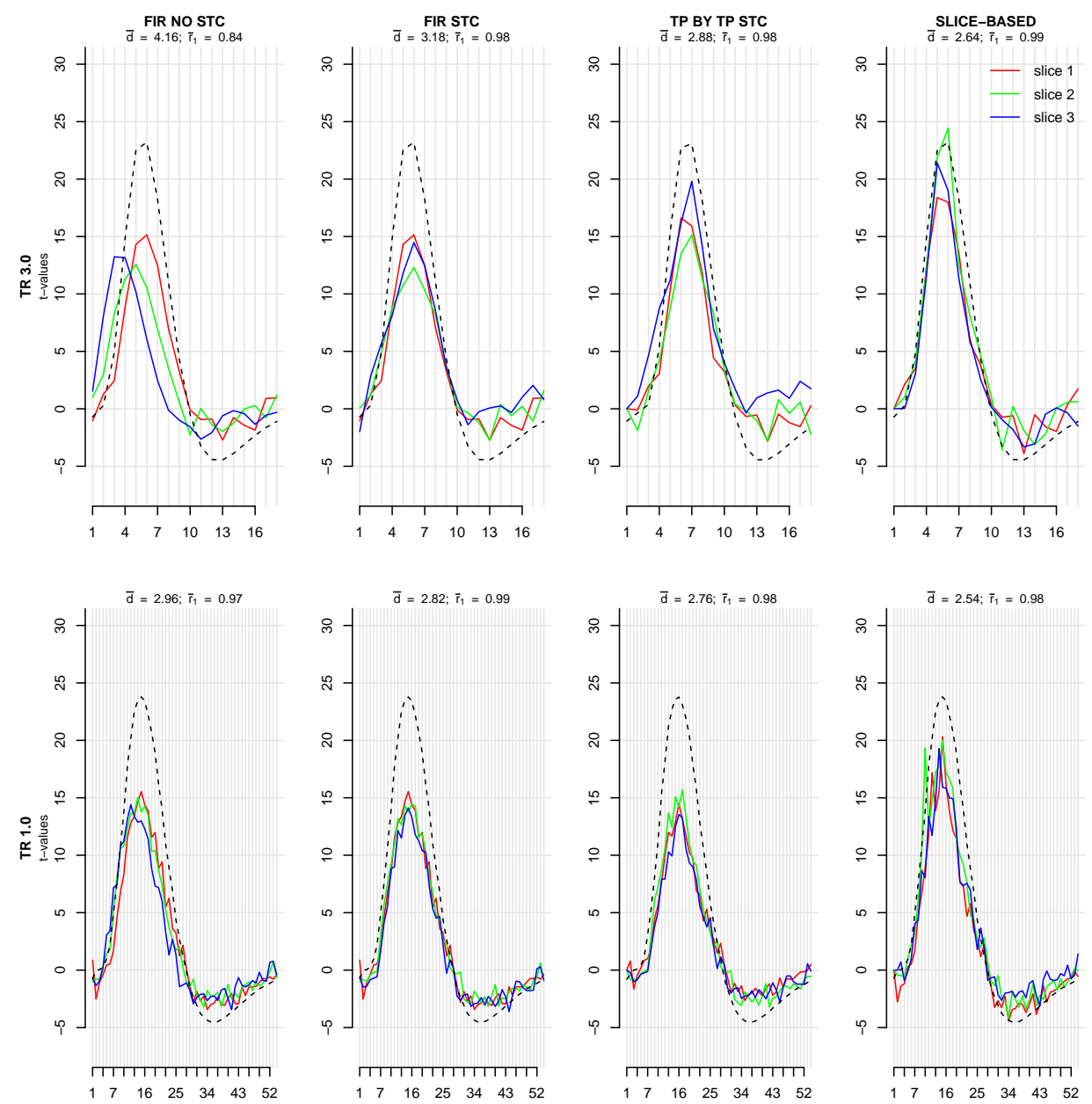

Figure 5: Differences in detection accuracy due to trial-by-trial variability in the shape of the BOLD signal in a simulated fMRI experiment. Half of the stimuli evoked a BOLD response with standard parameters, while the other half yielded a BOLD response with alternative parameters that indicated reduced dispersion of the main peak (see text for details). Note again that the Slice-Based method yielded the lowest $\bar{d}$ values, suggesting this method extracted the most similar ground-truth signal. 

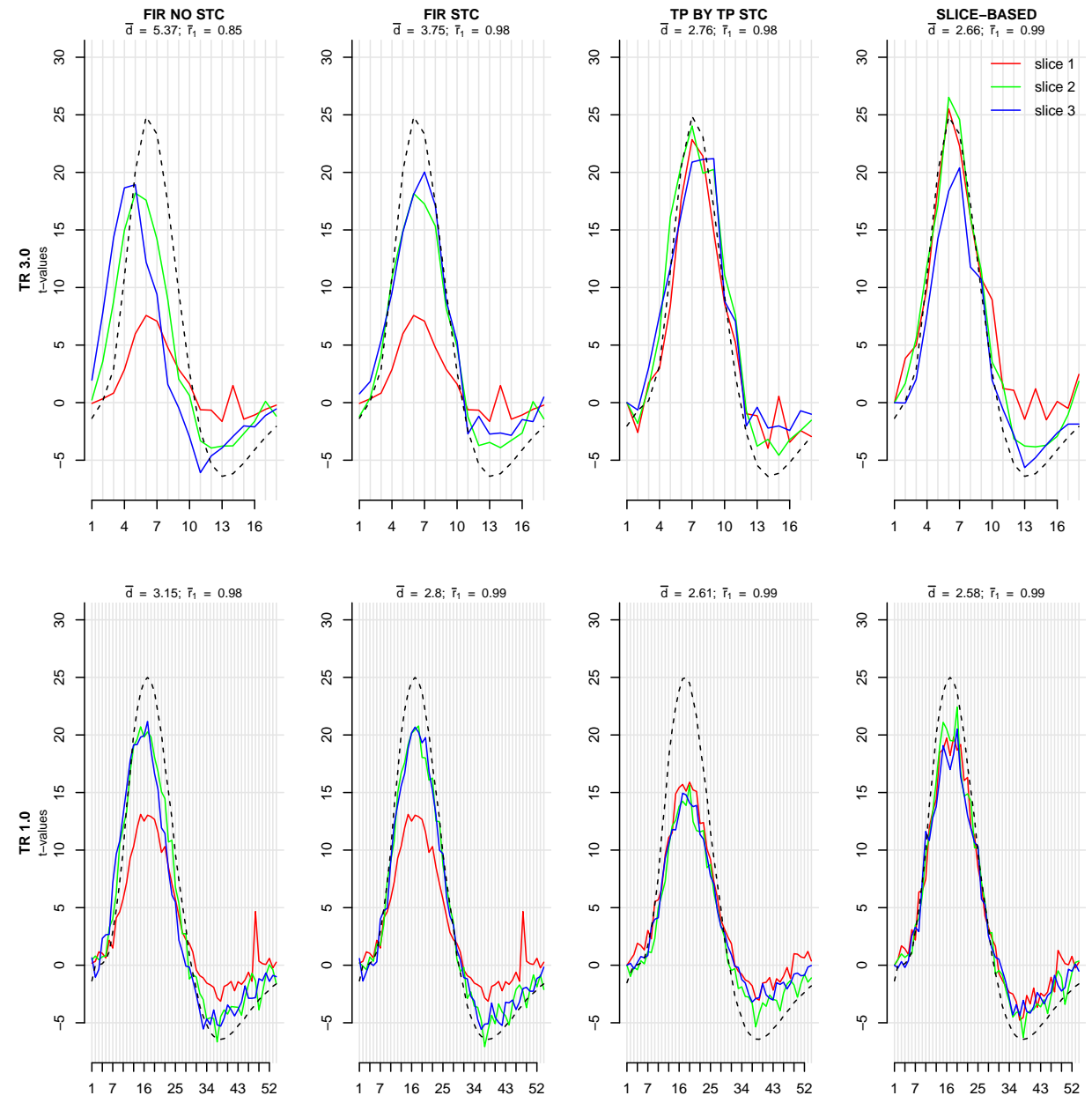

Figure 6: Differences in detection accuracy due to a single signal intensity spike in a simulated fMRI experiment. The single spike was modeled by changing a single intensity value in the time series sampled at the voxel on slice 1 (red line) to 5 times the maximum BOLD signal. The statistical impact of this single spike can be seen in the small peak in the undershoot of the extracted BOLD signal on slice 1. Note that this single spike strongly affected detection accuracy for the FIR based methods at all timepoints (note the overall reduced t-values for the red line), whereas detection accuracy was largely unaffected for the timepoint by timepoint methods. 

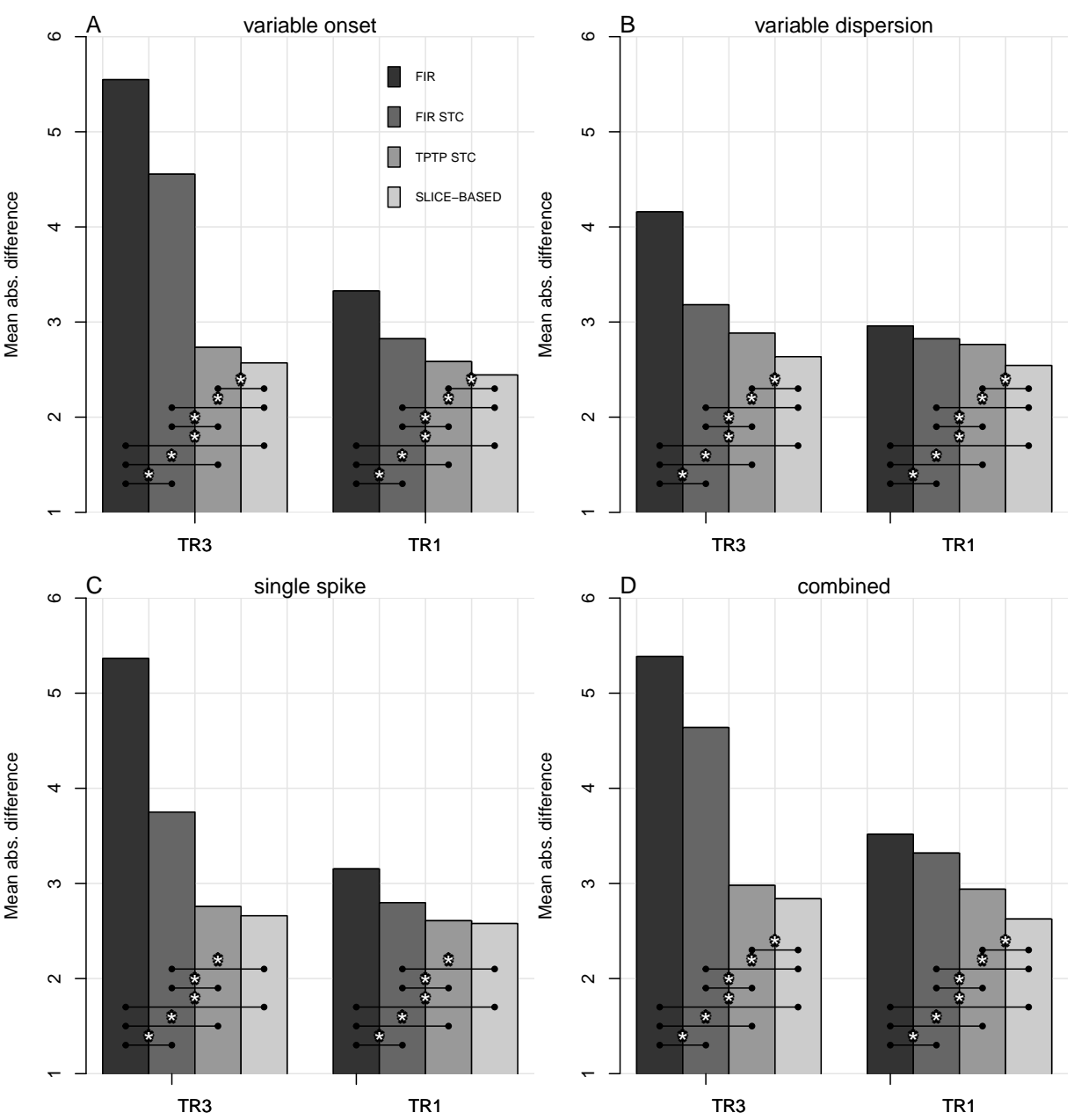

Figure 7: Graphical overview of the means and statistics of the three simulation experiments (panels A-C) and an additional simulation experiment combining all three previous simulations (panel D). Each bar represents the mean absolute difference between the ground-truth signal and the signal at each slice $(\bar{d})$. Note that for the Slice-Based method had the lowest $\bar{d}$ values, suggesting that the extracted signal more closely resembled the ground-truth signal. $\left(^{*}\right)$ denotes significant at $p_{i} 0.001$, see text for details. 


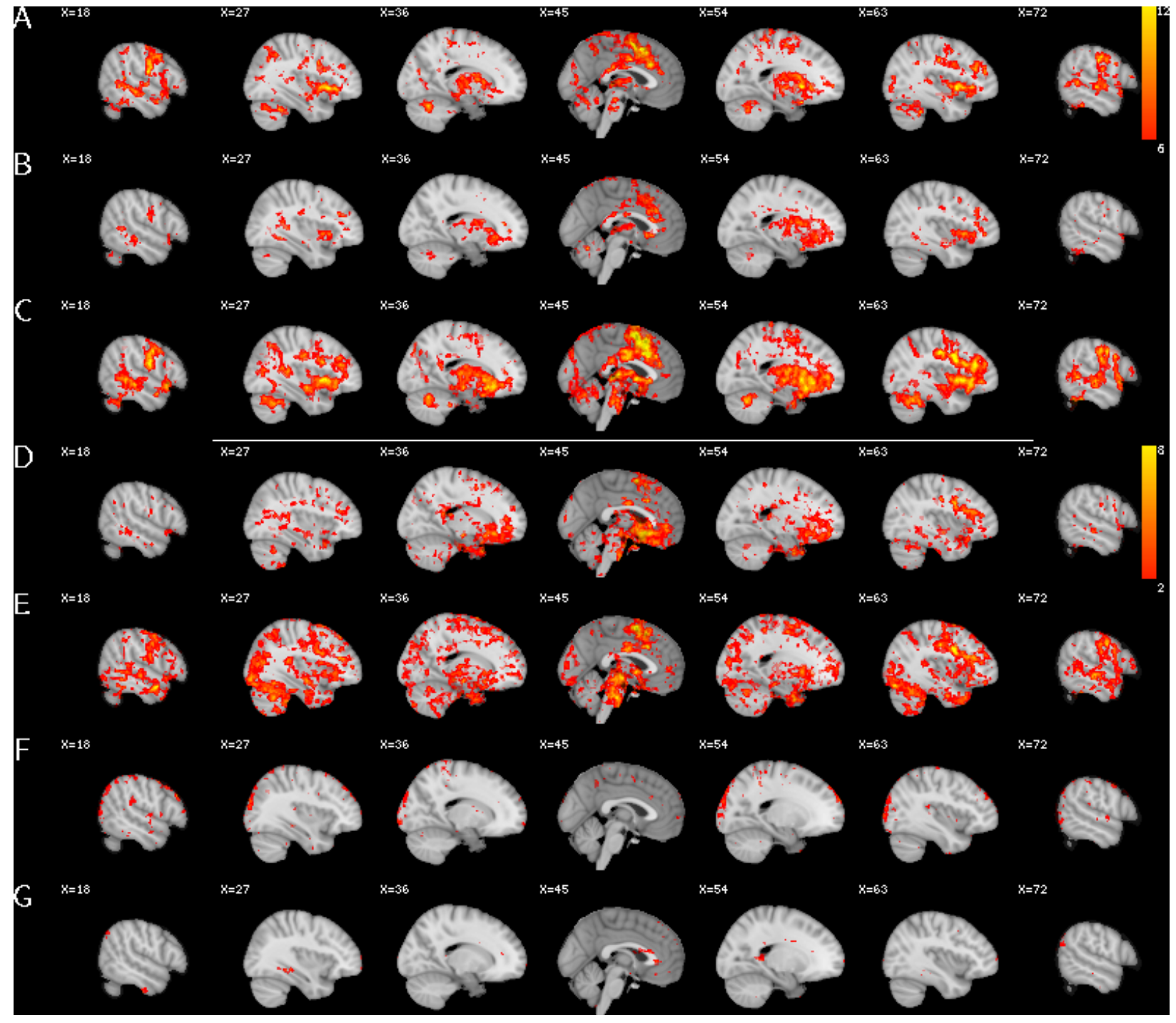

Figure 8: Comparison of standard GLM (panel A), Timepoint by Timepoint with STC (panel B) and Slice-Based (panel C) methods in basic signal detection during picture naming at the group-level with a threshold $t_{i} 6.0$. The same minimally preprocessed data was used for all three analyses (see text for details). Panels D-G reveal subtractions between unthresholded maps: Slice-Based minus GLM (panel D); Slice-Based minus Timepoint by Timepoint (panel E); GLM minus Slice-Based (panel F); Timepoint by Timepoint minus Slice-Based (panel G). Presented are saggital slices, slice number in upper left corner. Note that although all three methods yielded overall similar pattens of activity, the Slice-Based method has improved signal detection (most notably in medial frontal cortex, panels D and E). 
A

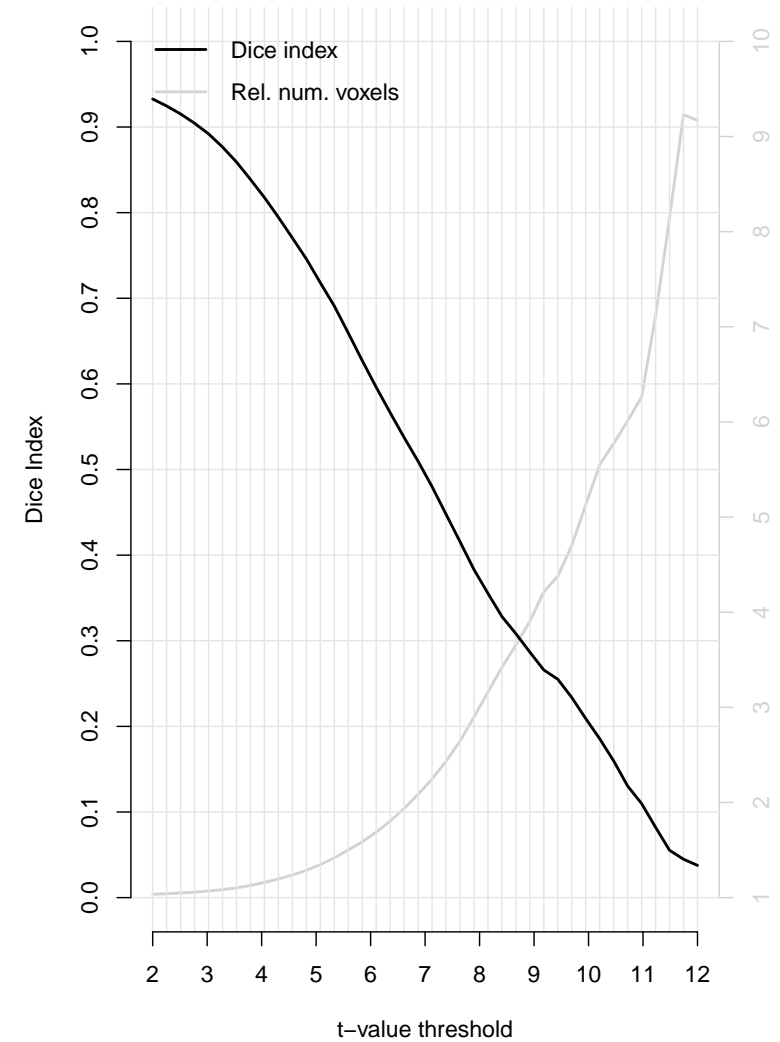

B

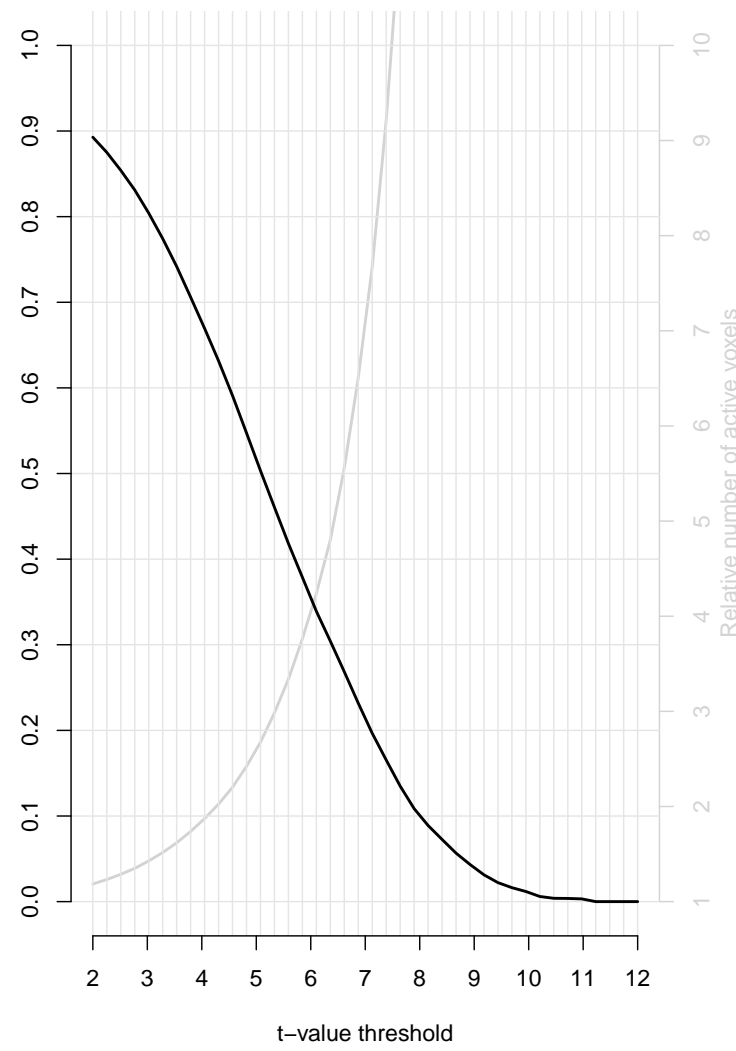

Figure 9: Similarity between the t-value maps of the standard Slice-Based method vs GLM (panel A) and Slice-Based vs Timepoint by Timepoint STC (panel B) at different $t$-value thresholds (x-axis). The lefthand y-axis shows the Dice index, an index of similarity between two statistical maps. The righthand $y$-axis shows the relative number of active voxels ( $\frac{\text { Slice Based }}{G L M}$ and $\left.\frac{\text { Slice Based }}{T P T P}\right)$. Note that the Dice index (black line) revealed decreased similarity between maps at higher thresholds $(t>4.0)$. Furthermore, this decreased similarity at higher thresholds is caused by a dramatic increase in active voxels in the Slice-Based map relative to the GLM and TPTP maps (grey line), suggesting improved signal detection for the Slice-Based method. 

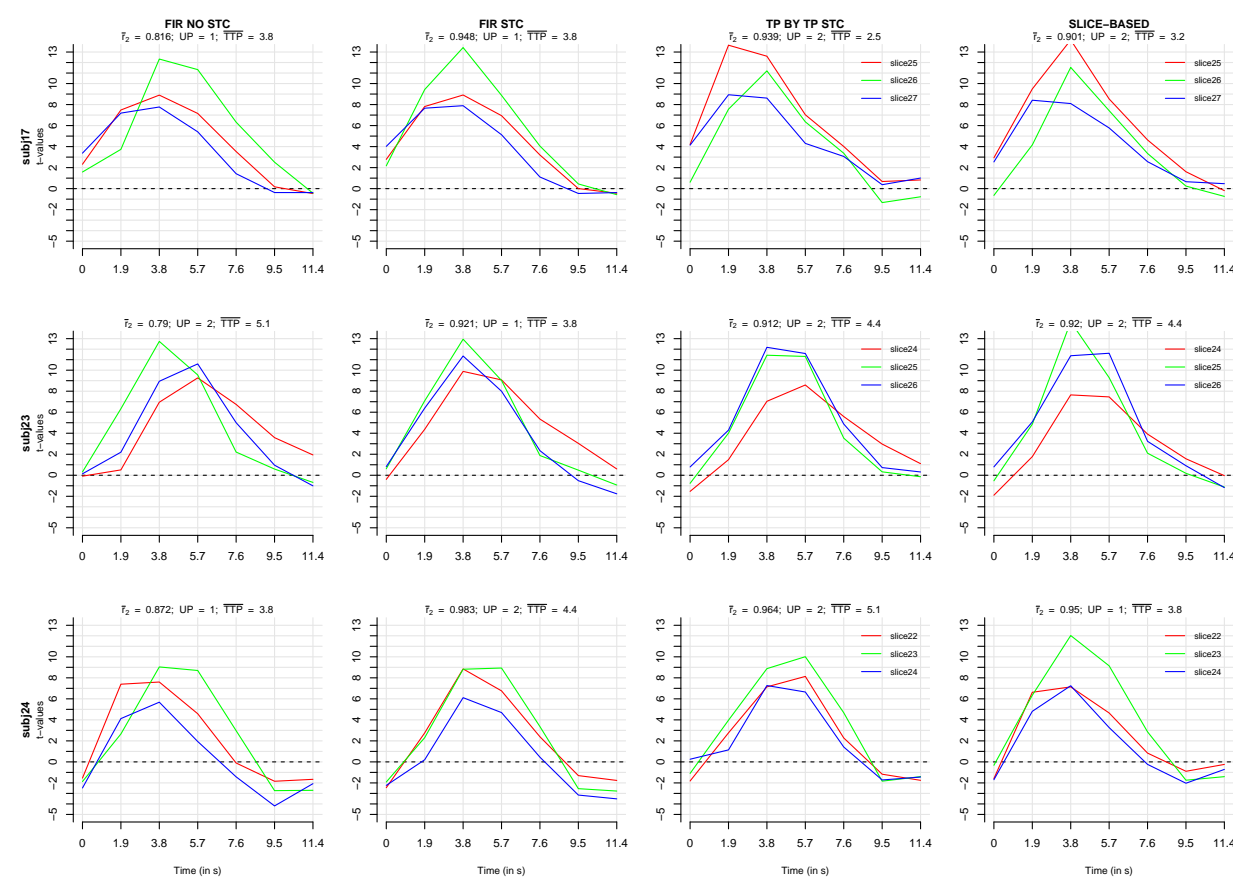

Figure 10: Method comparison using real data from left motor cortex activity obtained using the picture naming task. Each column in the figure represents a different method (first column = FIR without STC; second = FIR with STC; third = Timepoint by Timepoint with STC; fourth = Slice-Based). Signals are extracted from three voxels that appear on adjacent slices (see legend) in the left motor cortex in three representative subjects (top, middle, and bottom row for subjects 17, 23, and 24, respectively). Figure titles list the interslice correlation $\left(\bar{r}_{2}\right)$, the mean number of Unique Peaks (UP), and the mean Time To Peak (TTP) for the extracted signals in the graph. Note how the STC methods yielded smoother signals due to signal interpolation but had lower t-values than the Slice-Based method. 

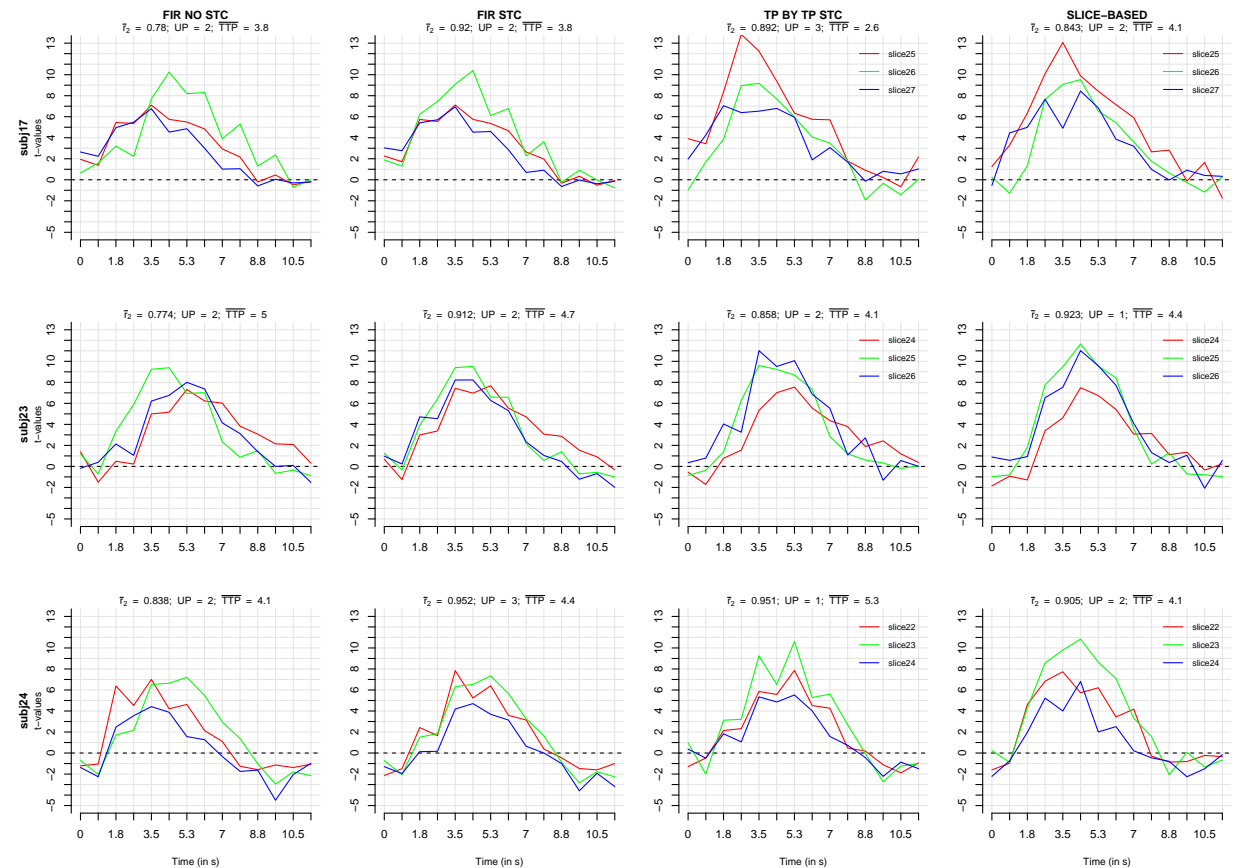

Figure 11: Method comparison using real data from left motor cortex activity obtained using the picture naming task. BOLD signal extracted using the four aforementioned methods at a fixed temporal resolution of 1/2 TR (954 ms). Other aspects identical to those used to obtain Figure 10 Not again how the Slice-Based method detected higher t-value signals despite the increase in temporal resolution. 

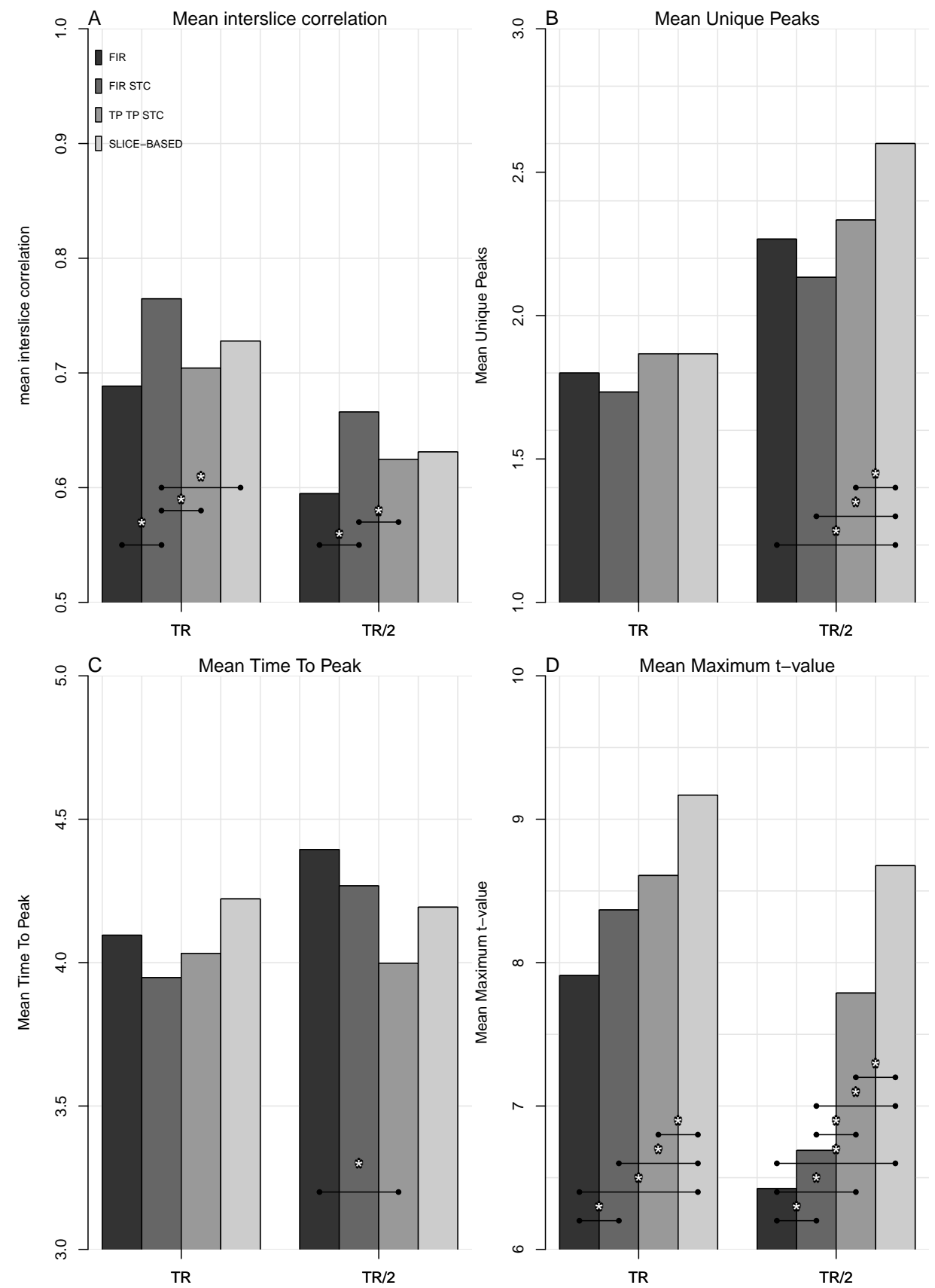

Figure 12: Mean interslice Pearson correlation (A), mean number of Unique Peaks (B), mean Time To Peak (C), and mean Max t-value (D) for the four methods at TR (1908 ms) and TR/2 (954 ms) temporal resolutions. Values obtained from three adjacent slices covering left motor cortex in 30 participants performing the picture naming task. $(*)$ denotes significant at $\mathrm{p} ; 0.05$. The slice-based method yielded increased t-values and more stable performance at higher temporal resolution. 